\title{
THE CHEMICAL COMPOSITION OF ASYMPTOTIC GIANT BRANCH STARS
}

\author{
David L. Lambert \\ Department of Astronomy, University of Texas, Austin, Texas
}

\begin{abstract}
Low resolution spectroscopic and photometric studies of Magellanic Cloud AGB stars have shown that the $M \rightarrow S \rightarrow C$ sequence is the result of the third dredge-up of ${ }^{12} \mathrm{C}$ and s-process elements in AGB stars of low mass. In this paper, data on the chemical compositions of normal Galactic $\mathrm{M} \rightarrow \mathrm{S} \rightarrow \mathrm{C}$ stars are reviewed and shown to be broadly consistent with expectations for the third dredge-up on the AGB.
\end{abstract}

\section{INTRODUCTION}

The title assigned to my talk was 'The Chemical Composition of Real PRG Stars'. A definition of what constitutes a 'real PRG star' was not provided except for the laconic comment 'not Barium stars'. For this review, I consider 'real PRG star' to be synonymous with 'asymptotic giant branch star' (AGB); i.e., a star with a C-O degenerate core, a thin He shell, and a deep H-rich convective envelope. The appearance of the adjective 'peculiar' in the title of this colloquium is intended, I suppose, in the context of a talk on chemical compositions to refer to stars having a non-solar mix of the chemical elements, but just such a mix is the fate of all AGB stars; they dredge up nuclear processed material on the ascent of the giant branch and again on the AGB. Abundance anomalies created by mass transfer or exchange across a binary system are outside my terms of reference.

Even with my definition of a real PRG star, the assigned topic is so broad that I must limit my remarks to two principal topics.

\section{Spectral Classification and Chemical Composition.}

A cursory inspection of the complex spectra of the late-type or PRG stars may convince the novice that extraction of chemical compositions is a burdensome and lengthy task. Fortunately, there are important indicators of chemical composition (for example, the $\mathrm{C} / \mathrm{O}$ ratio) that are obtainable by a mere inspection of low resolution spectra 
and, in some cases, of photometric indices. In other cases, examination of high resolution spectra may readily yield key information; the prime example is the presence or absence of technetium in AGB stars as judged by a search for the Tc I resonance lines in the blue. I comment on some of these possibilities for an "instant abundance analysis".

\section{Chemical Compositions of AGB Stars in the Sequence $M \rightarrow S \rightarrow C$.}

The label 'peculiar' seems inapposite when attached to these stars. The chemical compositions can now be determined with fair precision from high resolution optical and infrared spectra. Observed abundance differences with respect to the inferred initial (quasi-solar) compositions for these stars are attributable to mixing into the envelope of nucleosynthetic products from the He-burning shell. The principal products are carbon and those heavy elements synthesized by neutron capture through the s-process during a He-shell flash (thermal pulse). Although the observed and predicted changes of composition are not yet in full agreement, the measure of agreement surely suggests that these stars should no longer be marked out as 'peculiar'. I shall review recent explorations of the chemical compositions of stars in the sequence of increasing $C / O$ ratio: $M \rightarrow S \rightarrow C$.

After the $\mathrm{M} \rightarrow \mathrm{S} \rightarrow \mathrm{C}$ giants are culled from the lists of PRGs, there remains a mixed bag of peculiar stars whose evolutionary history is unclear. Examples include the remarkable star FG Sge, the hydrogen deficient carbon ( $\mathrm{HdC}$ ) stars, the R Coronae Borealis stars and especially the heavy element-enriched U Aqr, the warm carbon (R type) stars, the RV Tau and $\mathrm{W}$ Vir variables, and others. Some peculiar stars may be in a stage of rapid evolution experienced by the majority of AGB stars. Others may be following evolutionary paths avoided by the normal AGB stars. I shall make few references to peculiar giants.

\section{SPECTRAL CLASSIFICATION AND CHEMICAL COMPOSITION}

\subsection{The C/O Ratio}

Thanks to the preeminent role of the $\mathrm{CO}$ molecule in the dissociation equilibrium of carbon and oxygen, spectra of cool stars with an abundance ratio $\mathrm{C} / \mathrm{O}<1$ (here, oxygen-rich) are readily distinguishable at even low resolution from spectra of cool stars with an abundance ratio $\mathrm{C} / \mathrm{O}>1$ (here, carbon-rich). In the optical region, the spectra of oxygen-rich stars are dominated by metal oxide bands (e.g., TiO, $\mathrm{ZrO}, \mathrm{YO}$ ). In the same region, the spectra of carbon-rich stars are dominated by bands of $\mathrm{C}_{2}$ and $\mathrm{CN}$. Gross spectral differences between oxygen-rich and carbon-rich also exist in the infrared; 
cool oxygen-rich stars show $\mathrm{H}_{2} \mathrm{O}$ bands and carbon-rich stars show $\mathrm{C}_{2}$ and $\mathrm{CN}$ bands and, in the coolest stars, bands of polyatomic molecules such as $\mathrm{HCN}$ and $\mathrm{C}_{2} \mathrm{H}_{2}$.

Such gross spectral differences allow observers to classify stars according to the abundance ratio $\mathrm{C} / \mathrm{O}: \mathrm{C} / \mathrm{O}<1$ for oxygen-rich and $\mathrm{C} / \mathrm{O}>1$ for carbon-rich stars. A finer classification for the oxygen-rich stars has been attempted through observations of band ratios of two oxides. Since the molecular bands of $\mathrm{TiO}$ and $\mathrm{ZrO}$ are visible even at low dispersion, the ratio $\mathrm{ZrO} / \mathrm{TiO}$ is generally used. Although the principal reason for this ratio's dependence on O-C is well documented (see, for example, Wurm 1940 and Scalo 1974), misconceptions appear in the literature. Consider two metal oxides XO and ZO. If the molecular equilibrium for the metals involves only the competition between the metal and its oxide, it is readily shown that the partial pressure of the oxide is given by

$$
\mathrm{P}(\mathrm{XO})=\frac{\varepsilon_{\mathrm{X}} \mathrm{P}_{\mathrm{T}}(\mathrm{H}) \mathrm{P}(\mathrm{O})}{\left(\mathrm{K}_{\mathrm{XO}}+\mathrm{P}(\mathrm{O})\right)}
$$

where $\varepsilon_{X}$ is the abundance of $X$ relative to $H, P_{T}(H)$ is the "fictitious" total pressure of hydrogen $\left(\mathrm{P}_{\mathrm{T}}(\mathrm{H}) \simeq \mathrm{P}(\mathrm{H})+2 \mathrm{P}\left(\mathrm{H}_{2}\right)\right)$ and $\mathrm{K}_{\mathrm{XO}}$ is the equilibrium constant. Two limiting cases exist: (i) association of $X$ into $X O$ is nearly complete, then $P(X O)=\varepsilon_{X} P_{T}(H)$; (ii) the molecules $X O$ are a minor constituent, then $P(X O)=\varepsilon_{X} P_{T}(H) P(O) / K_{X O}$. Consider now the ratio $R=P(Z O) / P(X O)$. If case (i) applies to both molecules, $R=\varepsilon_{Z} / \varepsilon_{X}$. If case (ii) is appropriate for both molecules, $\mathrm{R}=\left(\varepsilon_{\mathrm{Z}} / \varepsilon_{\mathrm{X}}\right)\left(\mathrm{K}_{\mathrm{XO}} / \mathrm{K}_{\mathrm{ZO}}\right)$. These expressions are independent of the abundance difference (O-C) but depend, as expected, on the abundance ratio $\mathrm{Z} / \mathrm{X}$. A dependence on (O-C) arises when the molecule with the higher dissociation energy ( $\mathrm{ZrO})$ approaches case (i) and case (ii) is more appropriate for the other molecule (TiO). Then, $\mathrm{R}=\left(\varepsilon_{\mathrm{Z}} / \varepsilon_{\mathrm{X}}\right) \mathrm{K}_{\mathrm{XO}} / \mathrm{P}(\mathrm{O})$ and $\mathrm{P}(\mathrm{O}) \approx\left(\varepsilon_{\mathrm{O}}-\varepsilon_{\mathrm{C}}\right) \mathrm{P}_{\mathrm{T}}(\mathrm{H})$, i.e., $\mathrm{R} \propto(\mathrm{Z} / \mathrm{X}) /(\mathrm{O}-\mathrm{C})$. This case arises when the dissociation energies $D_{0}(X O)$ and $D_{0}(Z O)$ differ significantly, e.g., $\mathrm{D}_{\mathrm{o}}(\mathrm{TiO})=6.92 \pm 0.10 \mathrm{eV}$ and $\mathrm{D}_{\mathrm{o}}(\mathrm{ZrO})=8.00 \pm 0.14 \mathrm{eV}$ (Pedley and Marshall 1983). Of course, this simple argument ignores many factors: competition from metal dioxides and other molecules, changes in the atmospheric structure as the densities of major molecules change with the $\mathrm{O}-\mathrm{C}$ ratio, and variation of the molecular equilibrium with depth through the atmosphere. Nonetheless, it should be clear that $\mathrm{R}$ is controlled by $\mathrm{Z} / \mathrm{X}$ and, perhaps, also (O-C). For the (O-C) dependence to be strong, a star's effective temperature ( $\mathrm{T}_{\mathrm{eff}}$ ) and surface gravity (i.e., pressure) must be such that $\mathrm{Z}$ and $\mathrm{X}$ approach case (i) and case (ii) respectively in the photosphere. 
In defining type $S$ and introducing "an abundance class" defined by the relative intensities of $\mathrm{ZrO}$ and $\mathrm{TiO}$ bands, Keenan (1954) noted that "the questions of just which elements differ in abundance and of how large the differences are remain to be investigated on spectrograms of high dispersion". Ake (1979) introduced an abundance index defined by the relative intensities of TiO, $\mathrm{ZrO}$, and $\mathrm{YO}$ bands and calibrated the index in terms of the $\mathrm{C} / \mathrm{O}$ ratio by reference to synthetic spectrum calculations published by Scalo and Ross (1976). The $\mathrm{YO} / \mathrm{ZrO}$ ratio, which should be less sensitive to the metal abundances because $\mathrm{Y}$ and $\mathrm{Zr}$ are s-process elements, derives some of its $\mathrm{O}-\mathrm{C}$ sensitivity from the fact that dioxide formation is more important for $\mathrm{Y}$ than $\mathrm{Zr}$. Keenan and Boeshaar (1980), who classified $\mathrm{S}$ and $\mathrm{SC}$ stars using primarily $\mathrm{ZrO}, \mathrm{TiO}$ and $\mathrm{C}_{2}$ bands, also provide a $\mathrm{C} / \mathrm{O}$ index. These two abundance indices are assumed to be primarily controlled by the $\mathrm{C} / \mathrm{O}$ ratio. This assumption must derive in large part from the emphatic statements made by Scalo and Ross. Piccirillo (1980), who calculated molecular column densities for a grid of $\mathrm{M}$ and $\mathrm{S}$ model atmospheres, argues that the $\mathrm{ZrO} / \mathrm{TiO}$ strengthening seen in $\mathrm{S}$ stars "cannot be interpreted solely by $\mathrm{C} / \mathrm{O}$ effects" and "the observed strength of ZrO in the spectra of $S$ stars attests to $\mathrm{Zr}$ enhancement". These results from model atmosphere calculations confirm Boesgaard's (1970) demonstration that the abundance ratio $\mathrm{Zr} / \mathrm{Ti}$, as derived from a curve of growth analysis, is well correlated with a band index ZrO/TiO.

In short, the $\mathrm{ZrO} / \mathrm{TiO}$ band ratio is sensitive to both the $\mathrm{Zr} / \mathrm{Ti}$ and $\mathrm{O}-\mathrm{C}$ abundances. However, if the dredge-up of $\mathrm{C}$, the direct product of He-burning, and of $\mathrm{Zr}$, a s-process product, occur simultaneously, the $\mathrm{Zr} / \mathrm{Ti}$ at the surface will itself be a rough measure of the increased $\mathrm{C}$ abundance and, hence, the $\mathrm{ZrO} / \mathrm{TiO}$ ratio will indirectly through $\mathrm{Zr} / \mathrm{Ti}$ and directly through $\mathrm{O}-\mathrm{C}$ reflect the $\mathrm{O}-\mathrm{C}$ in the atmosphere. Today, we can calibrate the $\mathrm{ZrO} / \mathrm{TiO}$ vs $\mathrm{O}-\mathrm{C}$ dependence through abundance analyses of the brighter $\mathrm{M}$ and $\mathrm{S}$ stars (see Sec. 3.4). It should be possible using the $\mathrm{C}_{2}$ bands to extend the $\mathrm{O}-\mathrm{C}$ estimates to carbon stars. With a determined effort, it should be possible to calibrate Magellanic Cloud stars.

\subsection{Observation vs Theory: The Magellanic Clouds}

Searches of the Magellanic Clouds for carbon-enriched stars have yielded many new results on the evolution of AGB stars because reliable estimates of absolute luminosity are possible for Cloud members and the surface $\mathrm{C} / \mathrm{O}$ ratio may be obtained from inspection of low dispersion spectra. These "instant abundance analyses" are generally limited to assigning a star to one of three $\mathrm{C} / \mathrm{O}$ classes: $\mathrm{M}$ star with $\mathrm{C} / \mathrm{O} \leq \alpha, \mathrm{S}$ star with $\alpha$ $\leq \mathrm{C} / \mathrm{O}<1.00$, and $\mathrm{C}$ star with $\mathrm{C} / \mathrm{O}>1.00$ where $\alpha \simeq 0.95$ is usually assumed but our 
abundance analyses suggest a lower figure. Finer gradations are possible with the recognition of intermediate spectral classes and the calibration of an abundance index to MS and S stars. By searching the field and, in particular, the clusters in the Clouds for S and $C$ stars, critical information is obtained on the third dredge-up phase when freshly synthesized carbon and s-process elements from the He-burning shell enter the outer deep convective envelope.

About a decade ago, the theory of stellar evolution and nucleosynthesis on the AGB (Iben 1975, 1976, 1977; Truran and Iben 1977; Iben and Truran 1978) predicted the following:

(1) stars within the range 3 to $8 \mathrm{M}_{\odot}$ (the intermediate mass stars) become $\mathrm{C}$ stars and are the dominant supplier of s-process elements in the Galaxy and a major supplier of ${ }^{12} \mathrm{C}$. Significant dredge-up of ${ }^{12} \mathrm{C}$ and the s-process does not occur until luminosities of $\log \mathrm{L}_{\mathrm{L}} \odot \sim 4.3$ are reached. The s-process elements are produced in approximately their solar system proportions and in amounts such that intermediate mass stars account for the solar system ratio of s-process to iron peak elements. The neutron source is ${ }^{22} \mathrm{Ne}(\alpha, n)^{25} \mathrm{Mg}$ with the supply of ${ }^{22} \mathrm{Ne}$ controlled by the initial abundance of $\mathrm{C}$, $\mathrm{N}, \mathrm{O}$. The ability of the ${ }^{22} \mathrm{Ne}$ source to generate a solar system mix of heavy elements has been questioned (Busso et al. 1988), but these doubts depend greatly on the (uncertain) estimate of the ${ }^{22 \mathrm{Ne}}$ neutron capture cross section.

(2) stars less massive than about $2 \mathrm{M}_{\odot}$ do not become $C$ stars and stars less massive than about $3 \mathrm{M}_{\odot}$ do not ignite a neutron source and synthesize heavy elements by the s-process.

The stars in the Galaxy that are luminous enough to be on the AGB and whose atmospheres are enriched in ${ }^{12} \mathrm{C}$ and the s-process elements are the $\mathrm{S}$ stars and the $\mathrm{N}$-type carbon stars (Scalo 1976). Iben and Truran (1978) declined to recognize the abundance anomalies of these Galactic stars as being due to thermal pulses on the AGB. The two reasons cited were (i) the observed (uncertain) luminosities of $\mathrm{S}$ and $\mathrm{N}$ stars are below the predicted lower limit for dredge-up of ${ }^{12} \mathrm{C}$ by an intermediate-mass $\mathrm{AGB}$ star; (ii) the (qualitative) estimates of the Ba-peak to Sr-peak s-process abundances are too large to be consistent with production by the ${ }^{22 \mathrm{Ne}}$ source or compatible with the solar system abundances. Iben and Truran speculated on an alternative origin for $S$ and $N$ stars: "Perhaps the simplest explanation of S stars and of many $\mathrm{N}$-type carbon stars is that the surface composition characteristics originate during a helium [core] flash of an infrequently occurring nature". In such a core flash, the neutron source ${ }^{13} \mathrm{C}(\alpha, n)^{16} \mathrm{O}$ may be ignited 
with a neutron supply exceeding that generated by the ${ }^{22} \mathrm{Ne}(\alpha, \mathrm{n})^{25} \mathrm{Mg}$ reactions and, hence, higher $\mathrm{Ba} / \mathrm{Sr}$ ratios may be produced.

The predictions that dredge-up of ${ }^{12} \mathrm{C}$ and s-process was the sole prerogative of intermediate-mass AGB stars were first shaken and then shattered by surveys of cool carbon stars in the Magellanic Clouds. The luminosity function of $\mathrm{C}$ stars in the clouds peaks at $\mathrm{M}_{\mathrm{bol}} \sim-4.8$ or $\log \mathrm{L} / \mathrm{L}_{\odot} \sim 3.8$ (Cohen et al. 1981; Richer 1981). Carbon stars are not found with $\log \mathrm{L} / \mathrm{L}_{\odot}>4.3$ (Cohen et al. 1981; Wood, Bessell, and Fox 1983; Wood 1987); this luminosity was the predicted lower limit for carbon star production by intermediate mass stars. The low luminosity limit, $\left(\log L / L_{\odot} \sim 3.5\right)$ for the cool carbon stars just exceeds the luminosity achieved at the He core flash at the tip of the red giant branch. The observed luminosity range is consistent with an identification of cool carbon stars as thermally pulsing low mass AGB stars. The near absence of lower luminosity carbon stars suggests that the He core flash is not primarily responsible for carbon enrichment. Less luminous carbon stars exist, but appear to be counterparts of the Galactic warm (R-type) carbon stars whose origin appears unrelated to thermal pulsing in a single PRG.

Clearly, the observed luminosity function of cool carbon stars in the Clouds is in stark conflict with the theoretical predictions current in 1981: (i) oxygen-rich (M) stars are converted to carbon-rich (C) stars at a much lower luminosity and, hence, at a lower mass than predicted, and (ii) the intermediate mass stars predicted to become $C$ stars with $\mathrm{M}_{\mathrm{bol}} \leqslant$ -6 are either not formed or, if formed, either evolve much more rapidly than predicted (e.g., shed their envelope in a "superwind"), or are reconverted to an O-rich star. Resolution of this conflict ("The Carbon Star Mystery: Why Do the Low Mass Ones Become Such and Where have the High Mass Ones Gone?" - Iben [1981]) is discussed by Iben (1988) and in several papers at this colloquium.

Between the observed upper limit $\left(\mathrm{M}_{\mathrm{bol}} \sim-6\right)$ for cool carbon stars and the maximum luminosity for an AGB star $\left(\mathrm{M}_{\mathrm{bol}} \sim-7.1\right)$, a limit set when the degenerate core reaches the Chandrasekhar limit, the AGB stars in the clouds are oxygen-rich. This sample defined first by Wood, Bessell, and Fox (1983) contains a few S stars. Wood et al. speculate that $\mathrm{H}$-burning at the base of the convective envelope may through the cycling of $\mathrm{C}$ to $\mathrm{N}$ reconvert the carbon-star to an oxygen-star heavily enriched in nitrogen. Unfortunately, extraction of a $\mathrm{N}$ abundance for a cool oxygen-rich star calls for high resolution spectra or a thorough comparison of observed and synthetic low resolution spectra. 
Spectral classification of stars in the Magellanic Cloud clusters is providing even more detailed results on the evolution of AGB stars; e.g., the minimum mass for which He-shell flashes can convert a star to a carbon star: Bessell, Wood, and Lloyd Evans (1983) estimate $\mathrm{M}>0.9 \mathrm{M} \odot$ for $[\mathrm{Fe} / \mathrm{H}] \sim-1$ and $\mathrm{M}>1.3 \mathrm{M}_{\odot}$ for $[\mathrm{Fe} / \mathrm{H}] \sim 0$. The transition $\mathrm{M} \rightarrow$ $\mathrm{S} \rightarrow \mathrm{C}$ occurs at a higher luminosity and a lower $\mathrm{T}_{\text {eff }}$ in more massive stars (Lloyd Evans 1983, 1984; Frogel and Blanco 1984). Thorough scrutiny of individual AGB stars and the frequency of occurrence of $\mathrm{M}, \mathrm{S}$, and $\mathrm{C}$ stars in the clusters promises to provide critical challenges of the theory of PRGs; for example, Lloyd Evans (1984) uses Keenan and Boeshaar's (1980) ZrO index ("calibrated" by them in terms of the C/O ratio) to make the tentative suggestions that "the build-up in the $\mathrm{C} / \mathrm{O}$ value at the surface does not proceed at a uniform rate but accelerates abruptly after a slow start", "the S-star stage of evolution occupies a substantial period of time", "the observations are in better accord with an sprocess build-up which precedes the main change in the $\mathrm{C} / \mathrm{O}$ ratio". While "instant abundance analysis" of the Magellanic and other AGB stars will continue to provide novel results, more detailed abundance analyses of AGB stars in the Clouds and the Galaxy are surely necessary in order to complete the observational picture of the dredge-up of material from the He-burning shell.

In addition to insights into evolution on the AGB, low dispersion spectroscopy of Magellanic Cloud and Galactic stars yields cautionary hints; for example, Wood, Bessell, and Fox (1983) note that two SMC variables change from $S$ to $C$ stars as they vary. The atmospheric $\mathrm{C} / \mathrm{O}$ ratio is presumed to be unchanging, but it may be so close to unity that alterations to the atmospheric structure exert a controling influence on the partial pressure of carbon. These changes are reminiscent of longer-term trends from an oxygen-rich to a carbon-rich atmosphere reported for the Galactic stars TT Cen (Stephenson 1973) and BH Cru (Lloyd Evans 1985). Both are now SC stars but earlier showed ZrO bands. In these cases, the spectral classification appears to persist over several cycles.

\subsection{High Resolution Spectroscopy: Technetium}

Before I discuss some results obtained from rather detailed abundance analyses, I comment on extension of "instant abundance analyses" to high dispersion spectra. Undoubtedly the outstanding example is the detection of technetium through the Tc I resonance lines at 4238, 4262, and $4297 \AA$. Merrill's (1952) discovery of Tc in the atmospheres of $S$ stars is rightly a landmark in studies of PRGs. Tc is the outstanding indicator of recent and, perhaps, current s-process nucleosynthesis in PRGs. It is not 
always appreciated that (i) $\mathrm{Tc}$ is not omnipresent in a sample of stars enriched to similar levels in the heavy elements that are products of the same neutron capture s-process that is responsible for the synthesis of technetium; (ii) Tc is present in some stars for which overabundances of s-process elements appear to be very small.

Scalo and Miller (1981) first pointed out that, of 30 stars of type MS, S, SC, and C in a survey and compilation by Little-Marenin and Little (1979), 9 stars did not contain Tc. These spectral types exhibit, in general, s-process and ${ }^{12} \mathrm{C}$-enrichment. The absence of $\mathrm{Tc}$ in $30 \%$ of these stars led Scalo and Miller (1981) to suggest two possible explanations, both of which involved radical changes in the usual picture of AGB evolution: (i) the mixing of s-processed material to the surface does not occur at every shell flash - if many consecutive flashes occur without mixing into the envelope, the surface $\mathrm{Tc}$ decays steadily until it is replenished by a flash in which mixing occurs; and (ii) some stars above a certain core mass (say $\mathrm{M}_{\text {core }} \geq 0.8 \mathrm{M}_{\odot}$ ) never mix.

Iben and Renzini (1983) criticized Scalo and Miller's (1981) conclusions and suggested three alternative explanations for the Tc-poor stars. (1) Some stars may not be s-process enhanced; low-resolution classification spectra may be an inadequate basis from which to claim a definitive s-process enhancement. Indeed, we (Smith and Lambert 1986) found several stars classified as MS that did not exhibit measurable s-process enhancements. (2) The half-life of ${ }^{99} \mathrm{Tc}$ decreases from $2 \times 10^{5} \mathrm{yr}$ at low temperatures $\left(\mathrm{T} \leqslant 10^{8} \mathrm{~K}\right)$ to just $5 \mathrm{yr}$ at $\mathrm{T} \approx 3 \times 10^{8} \mathrm{~K}$, the expected temperature for the ${ }^{22} \mathrm{Ne}$ neutron source (Cosner and Truran 1981; Schatz 1983). Then, Tc's absence in some AGB stars might be understood if the s-process operated at temperatures so high that $\mathrm{Tc}$ is destroyed very quickly. However, this conclusion is not supported by detailed studies of s-processing during thermal pulses; Mathews et al. (1986) show that Tc is produced at the higher temperatures because higher neutron fluxes offset the higher decay rates of ${ }^{99} \mathrm{Tc}$ and, hence, the absence of $\mathrm{Tc}$ in a s-process enriched star is not strong evidence for a hot s-process site. (3) Some stars may be cooler (or evolved) examples of the $\mathrm{G}$ and $\mathrm{K}$ giant barium stars which also exhibit s-process enrichments, yet contain no Tc. Such cooler barium stars have not yet begun to dredge-up fresh additions of s-processed material including Tc. The discovery by McClure, Fletcher, and Nemec (1980) and McClure (1983, 1985), that probably all barium stars are binaries and may have white dwarf companions has led to the hypothesis that the barium stars are the result of a transfer of mass from an AGB star (now the white dwarf) to a donor (now the barium star). This mass transfer could have easily happened so long ago that the Tc has decayed away. 
When our recent survey (Smith and Lambert 1988a) for the TcI $4260 \AA$ line in $40 \mathrm{MS} / \mathrm{S}$ stars is combined with published reports on an additional 19 stars, we confirm that MS/S stars enriched in the s-process but not containing Tc are common: about $40 \%$ of the sample do not show Tc. A comparison of the (uncertain) space densities suggests that the majority of these s-process enriched stars lacking Tc are probably evolved Barium GK giants that have not yet begun to dredge-up carbon and s-process elements on the AGB. This proposal is open to a simple observational test: if the Tc-poor $S$ stars are descendants of Barium stars, they will show orbital radial velocity variations. Jorissen and Mayor (1988) observed $9 \mathrm{~S}$ stars with the CORAVEL spectrometer and report that at least 5 stars are binaries. An extension of this survey will test our proposal. If the test yields a negative result, it will be necessary to reconsider the Scalo and Miller (1981) ideas. Of course, as the M-type Barium stars evolve along the AGB, they will experience a dredgeup of additional carbon and s-processed material and presumably replenish their surface $\mathrm{Tc}$ abundance. However, the fraction of such stars among the total population of Tccontaining $S$ stars should be small. Clearly predictions about the dredge-up in single stars should be tested against observations of single stars not of evolved binary/Barium stars. "Instant abundance analysis" through a search of high resolution spectra for the TcI resonance lines enables us to identify the thermally pulsing AGB stars.

Other examples of "instant abundance analysis" from high resolution spectra include the isolation of Li-rich cool carbon and S stars (e.g., WZ Cas), the classification of the ${ }^{13} \mathrm{C}$ rich or J-type carbon stars, and the identification of the hydrogen deficient 'cool' carbon $(\mathrm{HdC})$ and the warmer $\mathrm{RCrB}$ variables.

\section{CHEMICAL COMPOSITION OF GALACTIC M, S, AND C STARS}

\subsection{Spectroscopic Tests of An Evolutionary Scenario}

Observations of the cool luminous stars in the Magellanic Clouds suggest that the regular or normal $\mathrm{M}, \mathrm{S}$, and $\mathrm{C}$ stars are AGB stars of low mass $\left(\mathrm{M} \sim 2 \mathrm{M}_{\odot}\right.$, say) in which He-shell flashes (thermal pulses) occur and products of nucleosynthesis in the He-burning shell are dredged to the surface. Through a progressive enrichment of ${ }^{12} \mathrm{C}$, the principal product of He-burning, the oxygen-rich $\mathrm{M}$ star is converted to an $\mathrm{S}$ star and then to a $C$ star; of course, if the ${ }^{12} \mathrm{C}$ enrichment from a single pulse is sufficiently severe, the star may jump from $\mathrm{M}$ or $\mathrm{MS}$ to $\mathrm{C}$ without an intermediate step as a $\mathrm{S}$ star. In these low mass stars, predicted temperatures in the He-burning convective shell are too low for 
the ${ }^{22} \mathrm{Ne}(\alpha, \mathrm{n})$ source to be the primary neutron source. The alternative neutron source for the lower temperatures is generally considered to be the ${ }^{13} \mathrm{C}(\alpha, n)^{16} \mathrm{O}$ reaction with an adequate supply of ${ }^{13} \mathrm{C}$ provided by a mixing or diffusing of protons into the ${ }^{12} \mathrm{C}$-rich $\mathrm{He}$ shell; a small admixture of protons leads to ${ }^{12} \mathrm{C}(\mathrm{p}, \gamma){ }^{13} \mathrm{~N}\left(\beta^{+}, v\right)^{13} \mathrm{C}$ and, if the protons are largely consumed in this chain, the ${ }^{13} \mathrm{C}$ avoids proton capture and is subsequently destroyed by ${ }^{13} \mathrm{C}(\alpha, \mathrm{n})^{16} \mathrm{O}$.

As the discussion of Tc in MS and S stars indicated, evolved Barium stars may be mistaken for single ('real') MS, S, and C stars. Barium stars are most probably created by mass transfer across a binary when an s-process enriched AGB (MS, S, or C) star sheds its envelope onto its companion, the present peculiar giant. Thanks to the mass transfer, the compositions of 'real' PRGs and the Barium stars must be similar; the abundance anomalies of the latter are expected to be diluted forms of those found in the MS, S, or C stars. Observational confirmation of this expectation has been presented (Lambert 1985, 1988). Spectroscopic tests of the proposal that single ('real') MS, S, and C stars are thermally pulsing low mass stars should be applied to samples cleansed of evolved Barium stars. While an attempt to isolate such samples is now being made, some of the tests described below involve samples which are likely to contain evolved Barium stars.

The spectroscopic tests are most simply introduced as a series of questions:

(1) Are the elemental and isotopic abundances of $C, N$, and $O$ in the $M, S$, and $C$ stars interpretable as a sequence in which ${ }^{12} \mathrm{C}$ is added in an increasing amount? If the base of the convective envelope is cool, the ${ }^{12} \mathrm{C}$ from He-burning will not experience H-burning (i.e., conversion of ${ }^{12} \mathrm{C}$ to ${ }^{14} \mathrm{~N}$ with a substantial amount of ${ }^{13} \mathrm{C}$ ) as it is dredged-up. Also, the He-burning shell is not expected to synthesize a significant amount of ${ }^{16} \mathrm{O}$. Then we expect (i) ${ }^{12} \mathrm{C} /{ }^{16} \mathrm{O}$ and ${ }^{12} \mathrm{C} /{ }^{13} \mathrm{C}$ to increase in a predictable way from $\mathrm{M}$ stars through $\mathrm{S}$ stars to $\mathrm{C}$ stars; (ii) the ${ }^{14} \mathrm{~N}$ and ${ }^{16} \mathrm{O}$ abundances should be similar along the $\mathrm{M} \rightarrow \mathrm{S} \rightarrow \mathrm{C}$ sequence; (iii) the oxygen isotopes ${ }^{17} \mathrm{O}$ and ${ }^{18} \mathrm{O}$ should show a smooth progression in which a modest decrease of ${ }^{18} \mathrm{O}$ and, perhaps, ${ }^{17} \mathrm{O}$ is expected in the $\mathrm{C}$ stars.

(2) Are the enhancements of the s-process elements in the S and C stars consistent with predicted dredge-up from layers exposed to neutrons released by the ${ }^{13} \mathrm{C}(\alpha, \mathrm{n})^{16} \mathrm{O}$ reaction? Or is the ${ }^{22} \mathrm{Ne}(\alpha, n)^{25} \mathrm{Mg}$ reaction the dominant neutron supplier? Close examination of the s-process products may reveal details of the average conditions at the s-process site; for example, the total exposure and the mean neutron density: are these 
quantities derived from observations consistent with predictions from model low-mass AGB stars? Are the AGB stars leading suppliers of s-process elements?

(3) Are there surprises in the observed chemical composition? Mild quantitative discrepancies between, for example, the predicted and observed $\mathrm{C} / \mathrm{O}$ ratios for $S$ stars of a given ${ }^{12} \mathrm{C} /{ }^{13} \mathrm{C}$ ratio are certainly a signal that revisions are necessary in the modeling of the thermal pulses or the abundance analyses or both. Such discrepancies are not surprises. They are an inevitable part of our current preliminary probing of these complex stars. A surprise is a major unexpected or rare signature in the chemical composition. A single example must here serve to illustrate my definition. Dominy and Wallerstein (1986) through empirical curves of growth derived the $\mathrm{V}, \mathrm{Zr}, \mathrm{Nb}$, and $\mathrm{Tc}$ abundances for the long-period variables $\chi \mathrm{Cyg}$ (known mild S star), o Cet, and R Lyr. For the traditional s-process, $\mathrm{Tc}, \mathrm{Nb}$, and $\mathrm{Zr}$ are predicted to be enhanced in abundance. The results for o Cet were a surprise: $\mathrm{Zr}$ has an approximately normal abundance, $\mathrm{Tc}$ is present (see also Little-Marenin and Little 1979), but $\mathrm{Nb}$ is depleted; i.e., the $\mathrm{Nb} / \mathrm{V}$ ratio is substantially - a factor of nearly 5 - less than solar. Dominy and Wallerstein account for the surprise by supposing a very small and recent $\left(<10^{5} \mathrm{yr}\right)$ pulse of neutrons converted the original ${ }^{93} \mathrm{Nb}$, the sole stable isotope, to ${ }^{94} \mathrm{Mo}$, and that this supply has not been replenished because the progenitor ${ }^{93} \mathrm{Zr}$ has a long half-life $\left(\tau_{1 / 2} \sim 1.5 \times 10^{6} \mathrm{yr}\right)$. An interesting task now is to examine additional stars for evidence of such mild pulses; a cobalt enrichment may be a more readily detected signature of such pulses (Smith and Lambert 1987). Long-period variables like o Cet with Tc but no reported overabundance of s-process elements are not rare (Little, Little-Marenin, and Bauer 1987). One may conjecture that such stars may be beginning that series of thermal pulses/shell flashes with which evolution on the AGB terminates. A reported abundance anomaly that fits my definition of a surprise is not always a true measure of the composition of a stellar atmosphere. Some surprises are the result of a defective analysis. I comment later on our earlier reports of anomalously low ${ }^{17} \mathrm{O}$ and ${ }^{18} \mathrm{O}$ abundances in $\mathrm{S}$ stars!

(4) Are all of the predicted phases of AGB evolution represented and then recognized in the sky? In posing this question, I am thinking of the proposal (Scalo, Despain, and Ulrich 1975; Renzini and Voli 1981) that the base of the convective envelope of very luminous AGB stars may be so hot that H-burning occurs such that carbon is converted to nitrogen, the $\mathrm{C} / \mathrm{O}$ ratio is reduced, and the carbon star is reconverted to an oxygen-rich star. This luminous oxygen-rich star would remain enriched in the s-process elements and, hence, would probably be classified as a S star rather than a M star. Stars with a 'hot bottomed convective envelope' (HBCE) have been promoted as the explanation for several odd stars; e.g., very N-rich planetary nebulae, and the Magellanic Cloud AGB 
Stars (oxygen-rich with a mixture of $S$ stars) with luminosities below the AGB limiting luminosity and above that of the most luminous cool carbon stars (see Sec. 2.2). To check these and other identifications with HBCE stars, it seems desirable to establish the primary signature of a $\mathrm{HBCE}$ - a high $\mathrm{N}$ abundance and a low ${ }^{12} \mathrm{C} /{ }^{13} \mathrm{C}$ ratio. A high s-process abundance would establish the star's credentials as a former thermally pulsing AGB stars. Perhaps the more massive stars such as the long-period variable supergiants identified by Wood, Bessell, and Fox (1983) in the Magellanic Clouds that do not experience thermal pulses may also develop a HBCE. Then, an s-process enrichment would not accompany the signatures of H-burning.

(5) Are the derived compositions of the $\mathrm{M}, \mathrm{S}$, and $\mathrm{C}$ stars consistent with those of closely related objects? I shall comment on the symbiotic stars where the relative $\mathrm{C}, \mathrm{N}$, and $\mathrm{O}$ abundances can be determined from the emission line fluxes. Since the hot emitting gas was recently shed by the $\mathrm{M}$ giant, the C/N/O ratios from the emission lines should be identical to those ratios derived from moleular absorption lines in the $\mathrm{M}$ giant's spectrum. Such a comparison of C/N/O ratios offers a valuable check on the consistency of the two quite different analyses. Another and similar comparison of $\mathrm{C}, \mathrm{N}$, and $\mathrm{O}$ abundances (now with respect to $\mathrm{H}$ ) is possible between the planetary nebulae and the $\mathrm{M}$, $\mathrm{S}$, and C stars.

\subsection{Carbon Enrichment: $M \rightarrow S \rightarrow C$}

Examination of the Magellanic Clouds and, in particular, of their clusters shows that the relative luminosities of stars in the $\mathrm{M} \rightarrow \mathrm{S} \rightarrow \mathrm{C}$ sequence are consistent with the idea that the sequence arises through the dredge-up of ${ }^{12} \mathrm{C}$ from a He-burning shell. The simplest working hypothesis is that (i) the dredge-up adds ${ }^{12} \mathrm{C}$ but not ${ }^{13} \mathrm{C}$ and $16 \mathrm{O}$ to the convective envelope; and (ii) the base of the envelope is cool so that hydrogen burning does not convert ${ }^{12} \mathrm{C}$ to ${ }^{13} \mathrm{C}$ and ${ }^{14} \mathrm{~N}$. On this hypothesis, the ${ }^{12} \mathrm{C} /{ }^{16} \mathrm{O}$ and ${ }^{12} \mathrm{C} /{ }^{13} \mathrm{C}$ ratios are predictable from the envelope's presumed composition prior to the dredge-up of ${ }^{12} \mathrm{C}$. This presumed composition is taken to be that of the $\mathrm{M}$ giants whose ${ }^{12} \mathrm{C},{ }^{13} \mathrm{C}$, and ${ }^{14} \mathrm{~N}$ abundances have been rearranged during the first (and, perhaps, the second) dredge-up as the star ascended the giant branch prior to He-core ignition. Although dredge-up of ${ }^{12} \mathrm{C}$ is now the widely accepted scheme for production of carbon stars, it was not so long ago that a competing nucleosynthetic scheme could not be distinguished on the basis of observations or theoretical plausibility - hydrogen burning at a high temperature converts ${ }^{12} \mathrm{C}$ and ${ }^{16} \mathrm{O}$ to ${ }^{14} \mathrm{~N}$ and achieves ${ }^{12} \mathrm{C} /{ }^{16} \mathrm{O}>1$ in equilibrium. Spectroscopic determinations of $\mathrm{C}, \mathrm{N}$, and $\mathrm{O}$ serve not only to check the working 
hypothesis about ${ }^{12} \mathrm{C}$ dredge-up, but also to eliminate competing schemes, including $\mathrm{H}$ burning.

In the $\mathrm{M}, \mathrm{S}$, and $\mathrm{C}$ stars, the $\mathrm{C}, \mathrm{N}$, and $\mathrm{O}$ abundances are obtainable only from molecular lines. For a complete set of molecules, it is necessary to obtain infrared spectra. In particular, the vibration-rotation (V-R) lines of the $\mathrm{CO}$ molecule's ground electronic state are the sole source of the $\mathrm{C}$ abundance in a cool oxygen-rich atmosphere and of the $\mathrm{O}$ abundance in a cool carbon rich atmosphere. Often, a simultaneous analysis of two or more molecules may be required; e.g., the OH V-R lines in the spectrum of an oxygen-rich star effectively measure the abundance difference $\mathrm{O}-\mathrm{C}$ so that $\mathrm{OH}$ and $\mathrm{CO}$ lines must be analysed simultaneously to yield the $\mathrm{O}$ and the $\mathrm{C}$ abundances. It is the development of high resolution infrared spectrometers that has made it possible to determine $\mathrm{C}, \mathrm{N}$, and $\mathrm{O}$ elemental and isotopic abundances for the AGB stars. The principal instrument has been the Fourier transform spectrometer at the KPNO $4 \mathrm{~m}$ telescope. Other critical factors behind recent analyses include the development and distribution of model atmospheres for both O-rich and C-rich stars, and the increasing availability and accuracy of the necessary basic molecular data. My discussion concentrates exclusively on the derived abundances; the methods of abundance analysis and the accuracy of the results are not discussed for reasons of space. The reader is urged to consult the primary references for the omitted and vital discussions.

For the oxygen-rich stars (M, MS, S), we determined the $\mathrm{C}, \mathrm{N}, \mathrm{O}$ elemental abundances primarily from the CO $1.6 \mu \mathrm{m}$ V-R, the OH V-R $1.6 \mu \mathrm{m}$, and the CN red system ( $\mathrm{A}^{2} \Pi-$ $\left.\mathrm{X}^{2} \Sigma, \Delta \mathrm{v}=-2\right) 2 \mu \mathrm{m}$ lines with NH V-R $3 \mu \mathrm{m}$ lines providing a check on the $\mathrm{N}$ abundance. The ${ }^{12} \mathrm{C} /{ }^{13} \mathrm{C}$, and ${ }^{16} \mathrm{O} /{ }^{17} \mathrm{O} / 18 \mathrm{O}$ ratios are determined from the $\mathrm{CO}$ lines. These model atmosphere high-resolution analyses of infrared spectra are described in detail by Smith and Lambert $(1985,1986,1988 b)$. For the cool carbon stars, I draw on our analysis of 30 stars (Lambert et al. 1986) in which the primary lines providing the elemental abundances were the CO $1.6 \mu \mathrm{m} V-R$, the $C_{2}$ Phillips $\left(A^{1} \Pi-X^{1} \Sigma^{+}, \Delta v=-2\right)$, and the $\mathrm{CN}$ red system $2 \mu \mathrm{m}$ lines - see our paper for a discussion of additional molecular transitions. The ${ }^{12} \mathrm{C} /{ }^{13} \mathrm{C}$ ratio was derived from the $\mathrm{CO} 1.6$ and $2.3 \mu \mathrm{m}$ and $\mathrm{CN}$ lines. The ${ }^{16} \mathrm{O} /{ }^{17} \mathrm{O} /{ }^{18} \mathrm{O}$ ratios were derived from the $\mathrm{CO}$ lines. To complete the sample of PRGs, I include the SC stars analysed by Dominy, Wallerstein, and Suntzeff (1986) and Dominy and Wallerstein (1987); note that the abundances of $C$ and $O$ were assumed to be equal for SC stars. 
Tsuji (1986), who obtained ${ }^{12} \mathrm{C}$ abundances for $18 \mathrm{M}$ giants from CO $2.3 \mu \mathrm{m}$ lines, reports lower abundances: $[\mathrm{C} / \mathrm{H}]=-0.65$, but our recent sample of $\mathrm{M}, \mathrm{MS}$, and S stars gives $[\mathrm{C} / \mathrm{H}]=-0.39$ for $\mathrm{M}$ stars and -0.17 for MS and $\mathrm{S}$ stars. Our value for the normal $\mathrm{M}$ stars is in fair agreement with the $\mathrm{C}$ deficiency of $\mathrm{K}$ giants $([\mathrm{C} / \mathrm{H}]=-0.33$, Lambert and Ries 1981). A contributing factor to the differences between our and Tsuji's $C$ abundances may be our use of CO $1.6 \mu \mathrm{m}$ lines and his use of CO $2.3 \mu \mathrm{m}$ lines - see Tsuji (this conference) who reports on a systematic difference between the $\mathrm{C}$ abundances derived fron weak 1.6 and $2.3 \mu \mathrm{m}$ lines. In our current sample, these abundances are quite similar except for a few stars for which the differences are up to 0.3 dex in the sense reported by Tsuji; i.e., the weak $2.3 \mu \mathrm{m}$ lines give the lower abundance. These exceptions are stars exhibiting $\mathrm{Tc}$ lines and, hence, may be more luminous than the rest of the sample. Results given here are based on the $\mathrm{CO} 1.6 \mu \mathrm{m}$ lines for the ${ }^{12} \mathrm{C}$ abundance and the ${ }^{12} \mathrm{C} /{ }^{13} \mathrm{C}$ ratio, and the $\mathrm{CO} 2.3 \mu \mathrm{m}$ lines for the $16 \mathrm{O} /{ }^{17} \mathrm{O} /{ }^{18} \mathrm{O}$ ratios.

The hypothesis that the progressive addition of ${ }^{12} \mathrm{C}$ controls the sequence $\mathrm{M} \rightarrow \mathrm{S} \rightarrow \mathrm{C}$ is checked in Figure 1. The $M$ giants define a small area in the ${ }^{12} \mathrm{C} /{ }^{16} \mathrm{O}$ vs ${ }^{12} \mathrm{C} /{ }^{13} \mathrm{C}$ plane and represent the initial composition at the start of the thermal pulsing stage on the AGB. Note that the scatter of the $\mathrm{M}$ giants is dominated by the observational errors so that the intrinsic spread must be smaller than shown. The displacement of the $\mathbf{M}$ giants from their initial solar-like $\left({ }^{12} \mathrm{C} / 16 \mathrm{O} \sim 0.6,{ }^{12} \mathrm{C} /{ }^{13} \mathrm{C} \sim 90\right)$ composition is in fair quantitative agreement with predictions for the first dredge-up occurring as a star ascends the giant branch prior to $\mathrm{He}$ core ignition. Furthermore, the location of the $\mathrm{M}$ giants in Figure 1 is shared by the $\mathrm{G}$ and K giants (Lambert and Ries 1981; Kjaergaard et al. 1982) which have also experienced the first dredge-up but have yet to evolve to the AGB. This coincidence of $G, K$, and $M$ giants is expected unless the $M$ giants contain stars sufficiently massive to have experienced the second dredge-up (Becker and Iben 1979). The agreement is pleasing because different sets of lines and model atmospheres are employed for the two groups of stars.

With the addition of ${ }^{12} \mathrm{C}$, the $\mathrm{M}$ giants would be displaced to the upper right in Figure 1. The solid lines describe the predicted change in composition as ${ }^{12} \mathrm{C}$ is added to the envelope of a $\mathrm{M}$ giant; the lines are labeled with the initial composition $\left({ }^{12} \mathrm{C} /{ }^{16} \mathrm{O},{ }^{12} \mathrm{C} /{ }^{13} \mathrm{C}\right)$ of the $\mathrm{M}$ giant. It is seen that the majority of the MS, S, SC, and $\mathrm{C}$ stars fall between these lines i.e., the ${ }^{12} \mathrm{C} /{ }^{16} \mathrm{O}$ and ${ }^{12} \mathrm{C} /{ }^{13} \mathrm{C}$ abundances are consistent with the hypothesis that these stars are evolved ${ }^{12} \mathrm{C}$-enriched $\mathrm{M}$ giants. The ${ }^{16} \mathrm{O}$ abundances show a very similar mean value and scatter for the three principal groups: $[\mathrm{O} / \mathrm{H}]=-0.18 \pm 0.02$ for the $\mathrm{M}$, 


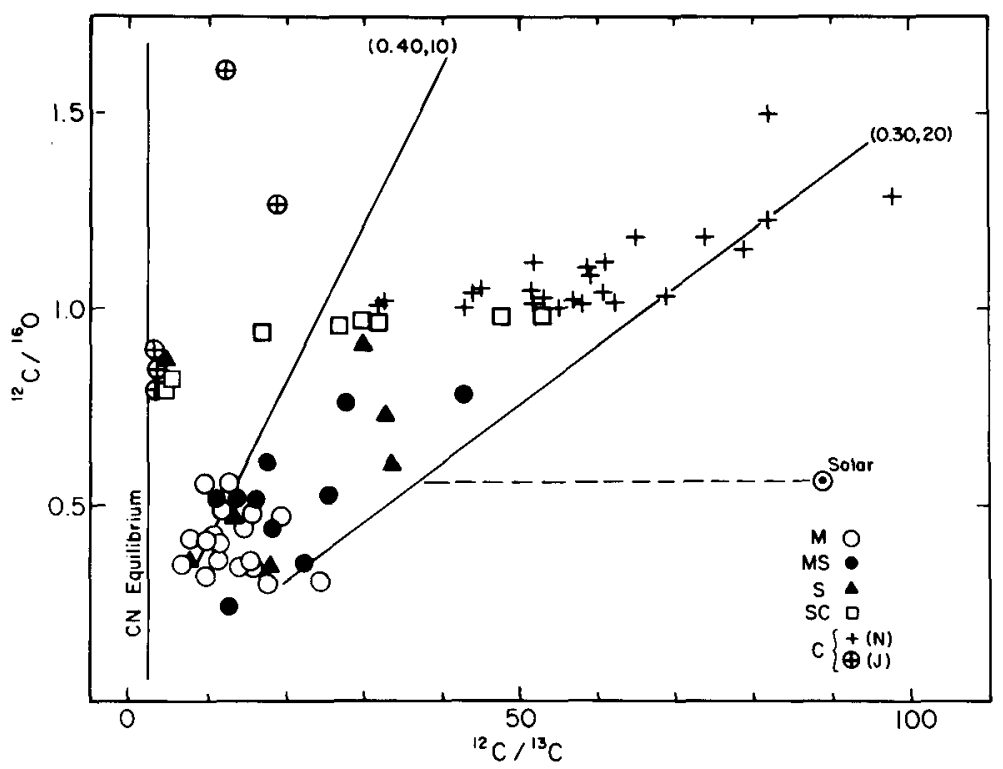

Figure $1 .{ }^{12} \mathrm{C} / 16 \mathrm{O}$ versus ${ }^{12} \mathrm{C} /{ }^{13} \mathrm{C}$ for cool giants. Note that the majority of the MS, $\mathrm{S}, \mathrm{SC}$, and $\mathrm{C}$ stars fall between the solid lines that trace the change in composition as ${ }^{12} \mathrm{C}$ is added to the envelope of a $\mathrm{M}$ giant; the lines are labeled with the initial composition $\left({ }^{12} \mathrm{C} /{ }^{16} \mathrm{O},{ }^{12} \mathrm{C} /{ }^{13} \mathrm{C}\right)$ of the $\mathrm{M}$ giant.

$\mathrm{MS}$, and $\mathrm{S}$ stars and $-0.22 \pm 0.03$ for the $\mathrm{C}$ stars. Hence, the differences in Figure 1 are due to ${ }^{12} \mathrm{C}$, not to an odd combination of ${ }^{12} \mathrm{C}$ and ${ }^{16} \mathrm{O}$ changes. A few stars fall outside the loci; note especially a MS, two $\mathrm{SC}$, and $3 \mathrm{C}$ stars with a ${ }^{12} \mathrm{C} /{ }^{13} \mathrm{C}$ ratio close to the equilibrium value for the $\mathrm{CN}$-cycle. Another obvious aspect (a surprise!) about Figure 1 is the absence of stars greatly enriched in ${ }^{12} \mathrm{C}$; i.e., the area between the loci and above ${ }^{12} \mathrm{C} / 16 \mathrm{O} \simeq 1.2$ is unoccupied. Scalo and Miller (1985, private communication) give frequency distributions for the $\mathrm{C} / \mathrm{O}$ ratio in simulated populations of $\mathrm{AGB}$ stars for various initial mass functions, birthrate histories, initial metallicity, and a simple representation of the dredge-up and mass loss. Although the observed distribution agrees quite well with the predicted one for the disk, this explanation would appear to be not the whole story because many $\mathrm{C}$-rich $\mathrm{PNs}$ have $\mathrm{C} / \mathrm{O}>1.2$. Other possible explanations include (i) the idea that carbon-rich envelopes may spawn copious amounts of graphite, which shroud the star, and hence, our sample may be biased toward low $\mathrm{C} / \mathrm{O}$ ratios; and (ii) more evolved or more massive $\mathrm{AGB}$ stars may dredge-up some 160 from the He shell and so flatten the predicted trend of ${ }^{12} \mathrm{C} / 16 \mathrm{O}$ with ${ }^{12} \mathrm{C} /{ }^{13} \mathrm{C}$.

If, as Figure 1 suggests, ${ }^{12} \mathrm{C}$ is added without significant exposure to the $\mathrm{CN}$-cycle $\mathrm{H}$ burning reactions, the ${ }^{14} \mathrm{~N}$ abundances should be similar along the $\mathrm{M} \rightarrow \mathrm{S} \rightarrow \mathrm{C}$ sequence. When three very $\mathrm{N}$-rich $\mathrm{S}$ stars are set aside, the $\mathrm{N}$ abundances for the $\mathrm{M}$ and the MS/S 
giants are quite similar; ${ }^{14} \mathrm{~N} / 16 \mathrm{O}=0.47 \pm 0.06$ from $18 \mathrm{M}$ giants and $0.45 \pm 0.06$ from 12 MS or S stars. However, as noted by Lambert $e t$ al. (1986), the cool carbon stars appear to be $\mathrm{N}$-deficient, not $\mathrm{N}$-rich as expected: $\langle[\mathrm{N} / \mathrm{H}]>=-0.27 \pm 0.05$ from a sample of 25 stars from which J-type stars are excluded. We suspect that systematic errors are responsible for this $\mathrm{N}$ deficiency. A possible source is the adopted $\mathrm{CN}$ dissociation energy and the $\mathrm{f}$-values of the red system. However, since the same $\mathrm{CN}$ lines are used in the analysis of the M, MS, and S stars, likely adjustments owing to the cooler temperatures of the $C$ stars appear to be small. Another candidate for the systematic error is the adopted $\mathrm{T}_{\text {eff }}$ scale for the $\mathrm{C}$ stars. If the $\mathrm{T}_{\text {eff' }}$ ' were raised, the $\mathrm{N}$ deficiency could be erased. Currently, we are determining accurate excitation temperatures for $\mathrm{CO}$ and $\mathrm{CN}$ from high quality infrared spectra, and careful calibration of the $T_{\text {exc }}-T_{\text {eff relation should provide a }}$ check on the $\mathrm{T}_{\mathrm{eff}}$ scale.
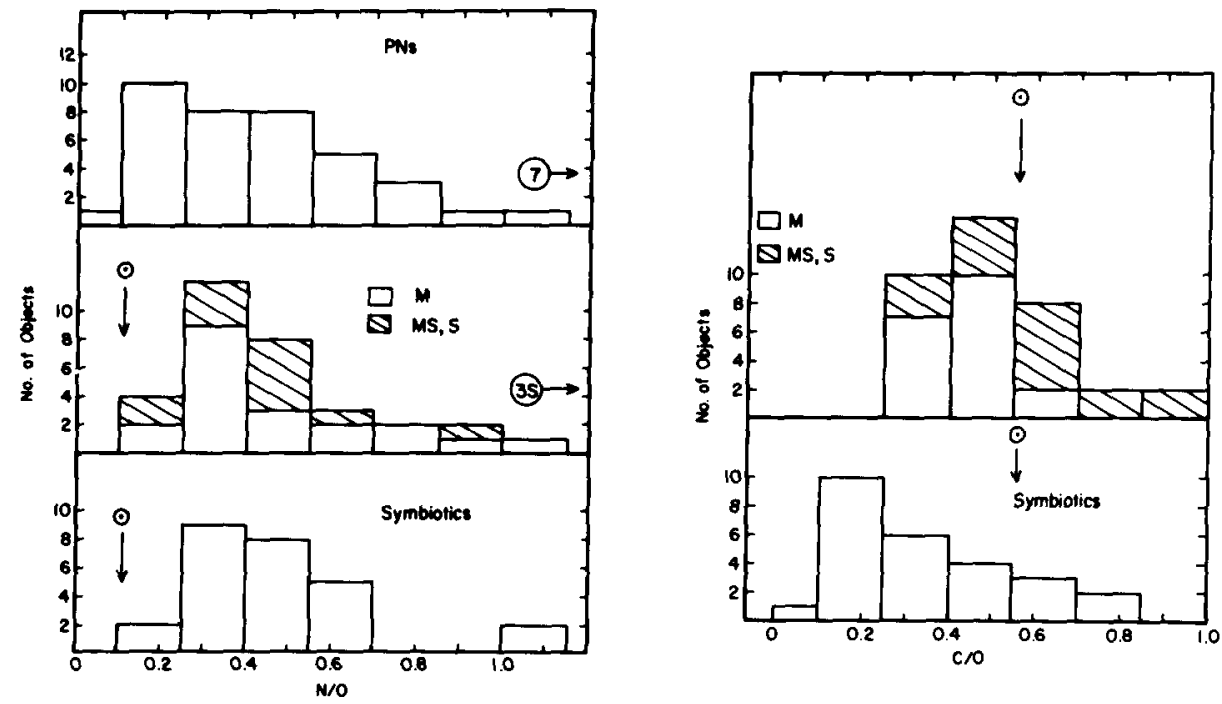

Figure 2 (left). - Distributions functions of N/O for symbiotic stars, AGB (M, MS, and $S$ ) stars, and planetary nebulae. Seven planetary nebulae and three $S$ stars are indicated as having $\mathrm{N} / \mathrm{O}>1.3$

Figure 3 (right). - Distribution functions of $\mathrm{C} / \mathrm{O}$ for symbiotic stars and AGB (M, MS, and S) stars.

Indications that the $\mathrm{N}$ abundance in $\mathrm{AGB}$ stars is more closely represented by the $\mathrm{M}$ and $\mathrm{S}$ stars than the $\mathrm{C}$ stars are provided through comparison with related objects. First, the $\mathrm{N}$ enrichment of $M$ and $S$ stars is similar, as expected, to that found for the $G$ and $K$ giants . Second, analyses of emission lines in ultraviolet spectra of symbiotic stars (Nussbaumer et al. 1988) and in ultraviolet and optical spectra of planetary nebulae (PN) (Aller and 
Czyzak 1983; Aller and Keyes 1987) confirm the N-enrichment - see Figure 2 for histograms compiled from the three samples. The sample of PN includes C-rich nebulae. For the three samples, the histograms are quite similar. A feature common to the MS/S and $\mathrm{PN}$ is the presence of very $\mathrm{N}$-rich objects: $\mathrm{N} / \mathrm{O}=4.0$ for the most $\mathrm{N}$-rich $\mathrm{S}$ star and $\mathrm{N} / \mathrm{O}=3.3$ for the extreme PN listed by Aller and Keyes (1987). The contrast with the C stars is especially striking when histograms are compared: the histogram (not illustrated!) for the $C$ stars would be confined entirely to the two leftmost boxes in Figure 2. In Figure 3, I show histograms of $\mathrm{C} / \mathrm{O}$ for the symbiotics and the $\mathrm{M}, \mathrm{MS}$, and $\mathrm{S}$ stars. Relative to the symbiotics, the stars seem to have systematically higher C/O ratios. If Tsuji's (1986) $\mathrm{C}$ abundances were substituted for the stars, the histograms would be quite similar. However, this substitution would result in higher stellar $\mathrm{N}$ abundances from the $\mathrm{CN} 2 \mu \mathrm{m}$ lines such that the N/O histograms (Figure 2) of the stars and the symbiotics would be dissimilar. Of course, these checks on the different analytical techniques (emission lines of ions in hot gas, absorption lines of molecules in a cool photosphere) should be taken to their obvious conclusion: we shall analyse infrared spectra of the $M$ stars in the symbiotics considered by Nussbaumer et al. (1988) who first drew attention to the similar C/N/O ratios!

Four $\mathrm{S}$ stars in the current sample are very $\mathrm{N}$-rich. Three are among our most extreme $\mathrm{S}$ stars: $[\mathrm{Y} / \mathrm{Fe}] \sim 0.7$ to 1.3 ; the analysis is incomplete for the fourth star. The star with the lowest N/O ratio of the quartet shows no Tc and, therefore, we suggest that it has not yet begun its series of He-shell flashes. The other three stars have yet to be examined for Tc. Since the sum of $\mathrm{C}, \mathrm{N}$, and $\mathrm{O}$ exceeds the initial sum by about a factor of 2 , it is likely that these stars have dredged up carbon and converted some of that carbon to nitrogen. However, the high ${ }^{12} \mathrm{C} /{ }^{13} \mathrm{C}(=12,30$, and 33$)$ ratios found in 3 stars appears at odds with this latter speculation. Perhaps we see these stars after the addition of fresh ${ }^{12} \mathrm{C}$ and before it is converted to ${ }^{14} \mathrm{~N}$ in the long interval between He-shell flashes. The exception, TV Aur with ${ }^{12} \mathrm{C} /{ }^{13} \mathrm{C}=5$, may possess the $\mathrm{HBCE}$ in which ${ }^{12} \mathrm{C}$ is converted to ${ }^{14} \mathrm{~N}$. These $\mathrm{N}$-rich stars, which are, perhaps, galactic counterparts of the luminous $\mathrm{S}$ stars discovered in the Magellanic Clouds by Wood, Bessell, and Fox (1983) are likely progenitors of the N-rich PN.

\subsection{The ${ }^{16} \mathrm{O} / 17 \mathrm{O} / 18 \mathrm{O}$ Ratios}

Since the oxygen isotopes react with hot protons and helium nuclei, the isotopic abundances in regions of $\mathrm{H}$ and $\mathrm{He}$ burning differ from those found in the gas from the star formed (here assumed to be those of solar system material: ${ }^{16} \mathrm{O} /{ }^{17} \mathrm{O}=2700$ 
and ${ }^{16} \mathrm{O} /{ }^{18} \mathrm{O}=490$. The initial abundances endure at the surface until the star begins its first ascent of the red giant branch. Then, the convective envelope extends downward into regions which underwent hydrogen burning via the $\mathrm{CNO}$ cycles during the main-sequence phase. The abundance of ${ }^{17} \mathrm{O}$ is enhanced by the CNO cycle reactions, so that after mixing, its abundance at the surface will be enhanced. The abundance of $18 \mathrm{O}$ in the interior is reduced slightly by ${ }^{18} \mathrm{O}(\mathrm{p}, \alpha)^{15} \mathrm{~N}$. At its maximum extent, the convective envelope may reach layers slightly depleted in $16 \mathrm{O}$. The carbon isotopes behave similarly to ${ }^{16} \mathrm{O}$ and ${ }^{17} \mathrm{O}$, with ${ }^{12} \mathrm{C}$ undergoing a rather larger depletion than $16 \mathrm{O}$, while ${ }^{13} \mathrm{C}$ is strongly enhanced by the $\mathrm{CNO}$ cycle like ${ }^{17} \mathrm{O}$ (and also ${ }^{14} \mathrm{~N}$ ). Characteristically, red giants in this phase of evolution exhibit ${ }^{12} \mathrm{C} /{ }^{13} \mathrm{C}$ ratios in the range $7-20$ (Figure 1 ), $16 \mathrm{O} /{ }^{17} \mathrm{O}$ ratios in the range $300-1000$, and ${ }^{16} \mathrm{O} /{ }^{18} \mathrm{O} \approx 500$ (Harris and Lambert 1984a,b; Harris, Lambert, and Smith 1988), in approximate accordance with these expectations. Early models predicted a larger ${ }^{18} \mathrm{O}$ depletion than observed, but prediction and observation are now in good agreement because, as suggested (Harris and Lambert 1984a,b), a new measurement has provided a lower rate constant for the ${ }^{18} \mathrm{O}(\mathrm{p}, \alpha)^{15} \mathrm{~N}$ reaction (Champagne and Pitt 1986). The predicted change in the ${ }^{16} \mathrm{O} /{ }^{17} \mathrm{O}$ ratio is dependent on mass. In low mass giants $\left(\mathrm{M}_{\varsigma} \leq 1.5 \mathrm{M}_{\odot}\right)$, the base of the convective envelope is not expected to reach the layers enhanced in ${ }^{17} \mathrm{O}$. In higher mass stars $\left(\mathrm{M} \gtrsim 3 \mathrm{M}_{\odot}\right)$, layers enhanced in $17 \mathrm{O}$ are mixed outwards and $16 \mathrm{O} / 17 \mathrm{O} \sim 500$ is predicted (see Harris, Lambert, and Smith 1988). For stars of 1.5 to $3 \mathrm{M}_{\odot}$ mass, the predicted ${ }^{16} \mathrm{O} /{ }^{17} \mathrm{O}$ runs from close to the initial value (2700) to 500. The predicted changes are sensitive to the rate of the key reaction ${ }^{17} \mathrm{O}(\mathrm{p}, \alpha)^{14} \mathrm{~N}$.

Following the end of core helium burning, a second dredge-up event is expected to occur in stars of above 4-5 M॰ (Iben and Renzini 1983). The convective envelope penetrates deeper down and into the hydrogen-exhausted region where the CNO cycle, burning in equilibrium, has transformed most of the original CNO nuclei in these layers into ${ }^{14} \mathrm{~N}$. Thus one effect of this second dredge-up is to enhance the ${ }^{14} \mathrm{~N}$ abundance at the surface over and above the enhancement occurring after the first dredge-up. Of the oxygen isotopes, ${ }^{16} \mathrm{O}$ and ${ }^{18} \mathrm{O}$ are little affected; their surface abundances are reduced slightly by this dilution of their post-first dredge-up abundances. Since the ${ }^{17} \mathrm{O}$ abundance rises to a peak between the layer reached at the first dredge-up and the deeper layer reached at the second dredge-up, the $16 \mathrm{O} / 17 \mathrm{O}$ ratio is expected to fall markedly after the second dredgeup. The predicted fall is sensitive to the rate adopted for ${ }^{17} \mathrm{O}(\mathrm{p}, \alpha)^{15} \mathrm{~N}$. Harris, Lambert, and Smith (1985) attempted to estimate the rate by matching predicted and observed ${ }^{16} \mathrm{O} /{ }^{17} \mathrm{O}$ ratios for $\alpha \mathrm{Her}$, a star that may have undergone a second dredge-up. It was concluded that the true rate for ${ }^{17} \mathrm{O}(\mathrm{p}, \alpha)$ is close to the "intermediate" rate of Fowler, Caughlan, and Zimmerman (1975). 
Shortly after entry onto the AGB (which coincides with the second dredge-up in sufficiently massive stars), low- and intermediate-mass stars undergo a succession of dredge-ups resulting from thermal pulsing, which penetrate down to layers exposed to $\mathrm{He}$ burning and which should be depleted of ${ }^{17} \mathrm{O}$. It is immediately apparent that the first such dredge-up must bring up all the material in which the ${ }^{17} \mathrm{O}$ abundance peak lies (if the second dredge-up has not already done so). Harris, Lambert, and Smith (1985) performed a similar calculation to that above for $\alpha$ Her, in order to obtain a prediction for the $160 /{ }^{17} \mathrm{O}$ following the onset of the third dredge-up process in AGB stars. ${ }^{16} \mathrm{O}$ and ${ }^{17} \mathrm{O}$ abundances were calculated for each zone of Becker and Iben's (1979) $3 \mathrm{M}_{\odot}(\mathrm{Y}=0.28, \mathrm{Z}=0.01)$ evolutionary model, estimating the third dredge-up depth from Iben and Renzini's (1983) heuristics. Using the "intermediate" rate for the reaction ${ }^{17} \mathrm{O}(\mathrm{p}, \alpha){ }^{15} \mathrm{~N}$, as established above from $\alpha$ Her data, the ratio after dredge-up was estimated at ${ }^{16} \mathrm{O} /{ }^{17} \mathrm{O} \approx 280$, compared with ${ }^{16} \mathrm{O} /{ }^{17} \mathrm{O} \approx 440$ before the third dredge-up. The ${ }^{16} \mathrm{O} /{ }^{18} \mathrm{O}$ ratios around 500 left by the first dredge-up are expected to be reduced slightly by the second dredge-up. The effect of the third dredge-up depends on how far the ${ }^{14} \mathrm{~N}$ left by the CNO cycle is processed to ${ }^{22} \mathrm{Ne}$ during helium burning. If the ${ }^{14} \mathrm{~N}$ is only burnt as far as ${ }^{18} \mathrm{O}$, then the ${ }^{18} \mathrm{O}$ abundance in the helium-burning region will be enhanced considerably. Stellar evolutionary model calculations suggest that in almost all cases the ${ }^{14} \mathrm{~N}$ is burnt to ${ }^{22} \mathrm{Ne}$ or ${ }^{25} \mathrm{Mg}$ (Becker and Iben 1979; Boothroyd and Sackmann 1988). If this is the case, no ${ }^{18} \mathrm{O}$ will be brought up by the third dredge-up, and the ${ }^{16} \mathrm{O} /{ }^{18} \mathrm{O}$ ratio will fall from $\sim 500$ to $\sim 600-700$ as the ${ }^{18} \mathrm{O}$ in the envelope is diluted by the ${ }^{18} \mathrm{O}$-free material brought up. If the reaction sequence upon ${ }^{14} \mathrm{~N}$ proceeds no further than ${ }^{18} \mathrm{O}$, a ratio ${ }^{16} \mathrm{O} /{ }^{18} \mathrm{O} \approx 100$ is possible.

Unfortunately, predictions of ${ }^{17} \mathrm{O}$ and ${ }^{18} \mathrm{O}$ for AGB models of stars of lower mass (say 2 $\mathrm{M}_{\odot}$ ) are not available; however, the ${ }^{16} \mathrm{O} /{ }^{17} \mathrm{O}$ ratio is expected to be somewhere between the $3 \mathrm{M}_{\odot}$ prediction and the maximum pre-third dredge-up ratio, i.e., $280 \leq{ }^{16} \mathrm{O} /{ }^{17} \mathrm{O} \leq$ 650. Since ${ }^{17} \mathrm{O}$ is not synthesized during the shell flashes, the material brought up by any subsequent dredge-up can only dilute the ${ }^{17} \mathrm{O}$ content of the envelope and increase the ${ }^{16} \mathrm{O} /{ }^{17} \mathrm{O}$ ratio. However, since the amount of material brought up is very small, this dilution effect should be very small. Unless destruction of ${ }^{14} \mathrm{~N}$ is halted at ${ }^{18} \mathrm{O}$, the $16 \mathrm{O} /{ }^{18} \mathrm{O}$ ratio is not expected to depart significantly from the pre-third dredge-up value of \%00). 
The ${ }^{17} \mathrm{O}$ and ${ }^{18} \mathrm{O}$ isotopic abundances are obtainable for AGB stars from the $2.3 \mu \mathrm{m} \mathrm{CO}$ lines. In the interval $4265-4295 \mathrm{~cm}^{-1}$, several $2-0 \mathrm{R}$-branch ${ }^{12} \mathrm{C}^{17} \mathrm{O}$ lines are readily

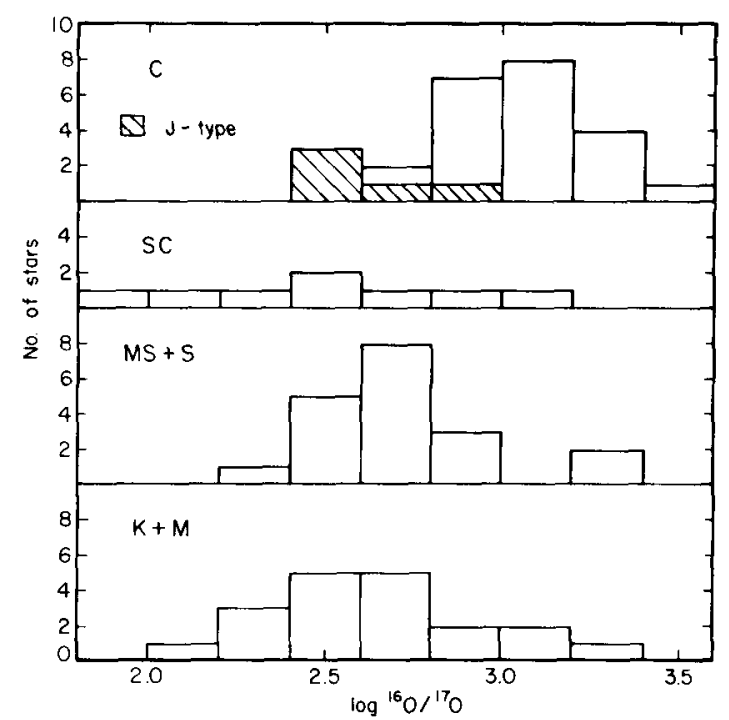

Figure 4. - Distribution functions of ${ }^{16} \mathrm{O} /{ }^{17} \mathrm{O}$ for samples of $\mathrm{K}$ and $\mathrm{M}, \mathrm{MS}$ and $\mathrm{S}, \mathrm{SC}$, and $\mathrm{C}$ stars.

detectable. Most lines are blended with $\mathrm{CN}$ red system lines, and overlying telluric lines must be ratioed out. There is not a comparably suitable region of ${ }^{12} \mathrm{C}^{18} \mathrm{O}$ lines. Our estimates of the ${ }^{16} \mathrm{O} /{ }^{18} \mathrm{O}$ ratio are based on syntheses of the region around the $2-0{ }^{12} \mathrm{C}^{18} \mathrm{O}$ $\mathrm{R}$-branch bandhead. A reliable determination of the ${ }^{16} \mathrm{O} /{ }^{17} \mathrm{O}$ or ${ }^{16} \mathrm{O} /{ }^{18} \mathrm{O}$ ratio requires that ${ }^{12} \mathrm{C}^{16} \mathrm{O}$ lines be matched approximately in strength with the ${ }^{12} \mathrm{C}^{17} \mathrm{O}$ and ${ }^{12} \mathrm{C}^{18} \mathrm{O}$ lines. This requirement is met with varying degrees of success through use of high excitation 2-0 $\mathrm{R}$-branch lines in the ${ }^{12} \mathrm{C}^{17} \mathrm{O}$ window.

The present discussion of AGB stars draws on isotopic ratios from Dominy, Wallerstein, and Suntzeff (1986) and Dominy and Wallerstein (1987) for SC stars, Smith and Lambert (1988b) for M, MS, and S stars, and Harris et al. (1987) for C stars. A sample of K and $M$ giants is drawn from Harris, Lambert, and Smith (1988). Oxygen isotopic ratios for these various samples are compared in Figures 4 and 5. Interpretation of the histograms of $160 /{ }^{17} \mathrm{O}$ determinations (Figure 4 ) is not a simple matter. Selection effects affect all the histograms. In addition, the $160 / \mathrm{O}^{17} \mathrm{O}$ ratio for the $\mathrm{K}$ and $\mathrm{M}$ giants affected by the first (and possibly the second) dredge-up is expected to depend on stellar mass. The most massive stars in this sample presumably do not become $\mathrm{S}$ and $\mathrm{C}$ stars. The less massive stars, 
which are left with a high ( $\sim$ initial) ${ }^{16} \mathrm{O} /{ }^{17} \mathrm{O}$ ratio following the first dredge-up, are predicted to show a lower ratio as AGB stars. An additional complication is that the MS, $S$, and $C$ samples likely contain evolved Barium stars in which the ${ }^{16} \mathrm{O} /{ }^{17} \mathrm{O}$ of the former AGB star (the mass donor in the binary) has been diluted with the ${ }^{17} \mathrm{O}$-poor material of the companion (the mass gainer and the present giant star) and then the ${ }^{16} \mathrm{O} /{ }^{17} \mathrm{O}$ of the mixture may have been further changed as the first dredge-up occurred in the Barium star. Perhaps one should draw just a single conclusion from Figure 4: the $\mathrm{C}$ stars (excluding the J-type) do appear to be deficient in ${ }^{17} \mathrm{O}$ relative to the MS, S, and SC stars. I hesitate to insist that this is real difference - see below.

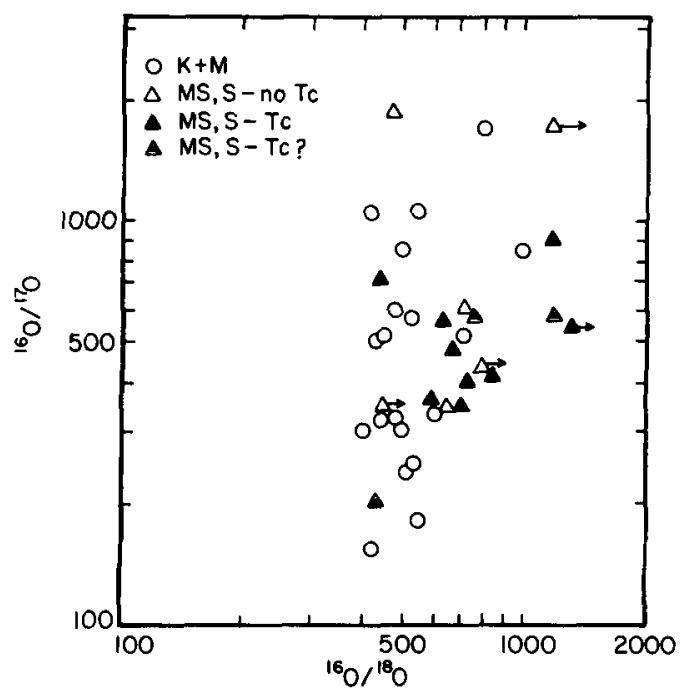

Figure 5. $-{ }^{16} \mathrm{O} /{ }^{17} \mathrm{O}$ vs ${ }^{16} \mathrm{O} /{ }^{18} \mathrm{O}$ for $\mathrm{K}, \mathrm{M}, \mathrm{MS}$, and $\mathrm{S}$ stars.

In Figure $5,160 / 17 \mathrm{O}$ and $160 / 18 \mathrm{O}$ ratios are compared for the samples of $\mathrm{K}, \mathrm{M}, \mathrm{MS}$, and $\mathrm{S}$ stars. ${ }^{18} \mathrm{O}$ abundances are not available for the $\mathrm{SC}$ stars in Figure 4 . The $\mathrm{K}$ and $\mathrm{M}$ stars span the predicted range in ${ }^{16} \mathrm{O} /{ }^{17} \mathrm{O}$ and, with few exceptions, show, as predicted, no significant depletion of ${ }^{18} \mathrm{O}$ below the presumed initial $(\simeq$ solar) ratio of $16 \mathrm{O} / 18 \mathrm{O}=490$. The exceptions, if real departures from the norm, may arise from mass loss or extensive mixing internal prior to the first or second dredge-up. On average, ${ }^{16} \mathrm{O} /{ }^{18} \mathrm{O}$ is reduced in the MS and S stars. In Figure 5, I distinguish stars with Tc from those without Tc; a search for Tc has yet to be attempted for four of the sample. The stars with Tc appear to be clustered near $16 \mathrm{O} / 17 \mathrm{O} \sim 500$ and cover a range in $16 \mathrm{O} /{ }^{18} \mathrm{O}$. Of the $\mathrm{SC}$ stars considered by Dominy, Wallerstein, and Suntzeff (1987) and Dominy and Wallerstein (1987), the two known to have $\mathrm{Tc}$ have ${ }^{16} \mathrm{O} /{ }^{17} \mathrm{O} \simeq 350$ and 200 . The present range in the ${ }^{16} \mathrm{O} /{ }^{17} \mathrm{O}$ ratio for the Tc stars which we identify as stars undergoing the third dredge-up is consistent with 
the approximate predictions discussed above, i.e., $280 \leq 16 \mathrm{O} /{ }^{17} \mathrm{O} \leq 650$. However, this tentative conclusion must be checked using a larger sample of MS and S stars. The observed ${ }^{16} \mathrm{O} /{ }^{18} \mathrm{O}$ ratios appear, in some cases, to be outside the predicted range of $16 \mathrm{O} /{ }^{18} \mathrm{O} \sim 500$ to 700 . However, when the approximate nature of the present predictions and the difficulty of extracting the ${ }^{18} \mathrm{O}$ abundance are considered, this discrepancy should not be overemphasized. Results for the MS and S stars without Tc should be considered in light of the mass transfer hypothesis; higher than typical $16 \mathrm{O} / 17 \mathrm{O}$ (and ${ }^{16} \mathrm{O} /{ }^{18} \mathrm{O}$ ) may be achievable by mixing of material from a $S$ star with unprocessed material of a normal star. The two possibly Barium-like giants in our recent sample (Smith and Lambert 1988b) with a high ${ }^{16} \mathrm{O} /{ }^{17} \mathrm{O}$ ratio are $\mathrm{HD} 35155$, the "neodymium" star (see below), and $\mathrm{HD} 96360$. The former is also ${ }^{18} \mathrm{O}$ deficient: ${ }^{16} \mathrm{O} / 18 \mathrm{O}>1200$. The sample of $\mathrm{SC}$ stars also includes one $\left(\mathrm{BD}+10^{\circ} 3764\right)$ with a high $16 \mathrm{O} /{ }^{17} \mathrm{O}(=1200)$ ratio; its status with respect to $\mathrm{Tc}$ is undetermined. When the $\mathrm{J}$ types are excluded, the carbon stars (Harris et al. 1987) define a fairly narrow inclined strip running from $\left({ }^{16} \mathrm{O} /{ }^{17} \mathrm{O},{ }^{16} \mathrm{O} /{ }^{18} \mathrm{O}\right) \simeq(600,900)$ to $(2500$, 2000). The shrouded carbon star IRC+10216 has $16 \mathrm{O} /{ }^{17} \mathrm{O} \simeq 650$ and $16 \mathrm{O} / 18 \mathrm{O} \simeq 875$ (Keady, Hall, and Ridgway 1988) according to analysis of the CO absorption lines produced in the circumstellar shell. This (typical?) carbon star with ${ }^{12} \mathrm{C} /{ }^{13} \mathrm{C} \sim 35$ falls among the $S$ stars in Figure 5 and at one end of the distribution of cool carbon stars.

The extreme ratios of ${ }^{16} \mathrm{O} /{ }^{17} \mathrm{O} \sim{ }^{16} \mathrm{O} /{ }^{18} \mathrm{O} \sim 1000$ to 2000 suggested for the cool carbon stars are difficult to explain in terms of evolution on the AGB - see Harris, Lambert, and Smith (1985, 1988) and Harris et al. (1988) for a discussion. Indeed, I would term the high ratios "a surprise" - see Sec. 3.1. Dominy and Wallerstein's (1987) reanalyses of two S stars gave much lower ratios than our previously obtained high ratios. Subsequently we have obtained new higher-quality spectra of several stars and analysed the ${ }^{12} \mathrm{C}^{17} \mathrm{O}$ and ${ }^{12} \mathrm{C}^{18} \mathrm{O}$ lines with respect to both ${ }^{12} \mathrm{C}^{16} \mathrm{O}$ high excitation $2.3 \mu \mathrm{m}$ lines and second-overtone lines at $1.6 \mu \mathrm{m}$. Several stars have now been analysed independently. Table 1 shows that consistent results can be achieved. Our "new and improved" results as well as those of several additional MS and $\mathrm{S}$ stars show that the ${ }^{16} \mathrm{O} /{ }^{17} \mathrm{O}$ and ${ }^{16} \mathrm{O}^{118} \mathrm{O}$ ratios span a narrower range than we previously suggested; the previous extreme was $16 \mathrm{O} /{ }^{17} \mathrm{O}=3000(-1200,+2500)$ for $\mathrm{HR} 363$ (a star not yet reanalysed) and the current extreme is ${ }^{16} \mathrm{O} /{ }^{17} \mathrm{O}=1900 \pm 600$ for $\mathrm{HD} 96360$, an $\mathrm{S}$ star without Tc. This narrower range is probably within predicted limits. In light of this experience, we are presently reanalysing some of the $\mathrm{C}$ stars using higher quality spectra. 


\section{TABLE 1}

Comparison of $160 / 17 \mathrm{O}$ Ratios

\begin{tabular}{|c|c|c|c|}
\hline Star & Type & $16 \mathrm{O} /{ }^{17} \mathrm{O}$ & Reference \\
\hline \multirow[t]{2}{*}{$\alpha \operatorname{Her}$} & M5II & $\begin{array}{l}190 \pm 80 \\
200 \pm 25^{\mathrm{a}}\end{array}$ & $\begin{array}{l}\text { Tsuji (1985) } \\
\text { Harris and Lambert (1984b) }\end{array}$ \\
\hline & & $180 \begin{array}{c}-50^{b} \\
+70\end{array}$ & \\
\hline \multirow[t]{2}{*}{ HR 1105} & S3.5 & $500 \begin{array}{c}+300 \\
-100\end{array}$ & Dominy and Wallerstein (1987) \\
\hline & & $350_{-100}^{+150^{c}}$ & Smith and Lambert (1988b) \\
\hline \multirow[t]{2}{*}{ HR 8062} & S4 & $930 \begin{array}{c}+400 \\
-200\end{array}$ & Dominy and Wallerstein (1987) \\
\hline & & $500+200^{d}$ & Smith and Lambert (1988b) \\
\hline \multirow[t]{2}{*}{ WZ Cas } & $\mathrm{SC} 7$ & $465_{-100}^{+150}$ & Dominy and Wallerstein (1987) \\
\hline & & $400_{-100}^{+175}$ & Harris et al. (1987) \\
\hline
\end{tabular}

a Based on the $2.3 \mu \mathrm{m}$ CO lines.

$\mathrm{b}$ Based on the fundamental $5 \mu \mathrm{m} \mathrm{CO}$ lines.

${ }^{c}$ Previous results were ${ }^{16} \mathrm{O} /{ }^{17} \mathrm{O}=2250(+700,-550)$ by Harris, Lambert, and Smith $(1985)$, and 600 $(+200,-150)$ by Harris et al. (1987).

d Previous result was ${ }^{16} \mathrm{O} /{ }^{17} \mathrm{O}=1850(+550,-425)$ by Harris, Lambert, and Smith (1985).

\subsection{The s-process}

This discussion of the s-process enrichment of MS and S stars draws on our recent analyses (Smith and Lambert 1985, 1986, 1988b) in which intervals in the nearinfrared (7400-7580 $\AA, 9980-10100 \AA$ ) were observed at a resolution of $0.2 \AA$. In all except the coolest stars, these intervals appear to be free from molecular line blanketing. Available atomic lines provide a useful selection of elemental abundances (e.g., $\mathrm{Ti}, \mathrm{Fe}, \mathrm{Ni}$, $\mathrm{Sr}, \mathrm{Y}, \mathrm{Zr}, \mathrm{Ba}$, and $\mathrm{Nd}$ ). The iron-peak abundances generally fall, as expected, in the range $[\mathrm{Fe} / \mathrm{H}]=-0.2$ to +0.2 . Of the s-process elements, $\mathrm{Y}, \mathrm{Zr}$, and $\mathrm{Nd}$ are the most reliably determined. This trio provides a measure of the overall overabundance of $s$ process elements and the level of exposure to neutrons. 
The overabundance as expressed by the mean of [Y/Fe] and $[\mathrm{Nd} / \mathrm{Fe}]$ is correlated with the carbon enrichment $\left(\left[{ }^{12} \mathrm{C} / \mathrm{Fe}\right]\right)$ - see Figure 6 . This correlation suggests that carbon and sprocess synthesis are intimately related as is required by models of thermally pulsing AGB stars. An evolutionary relation between $M, M S, S$, and $C$ stars is suggested by the fact that, in Figure 6, the MS and S stars connect the M to the N-type C stars. By contrast, the J-type $\left({ }^{13} \mathrm{C}\right.$-rich) $\mathrm{C}$ stars and the warm or R-type carbon stars are carbon rich but not significantly s-process enriched. A calibration of the "abundance" index appended to the spectral type of MS and S stars - see Sec. 2.1 - could now be provided from Figure 6.

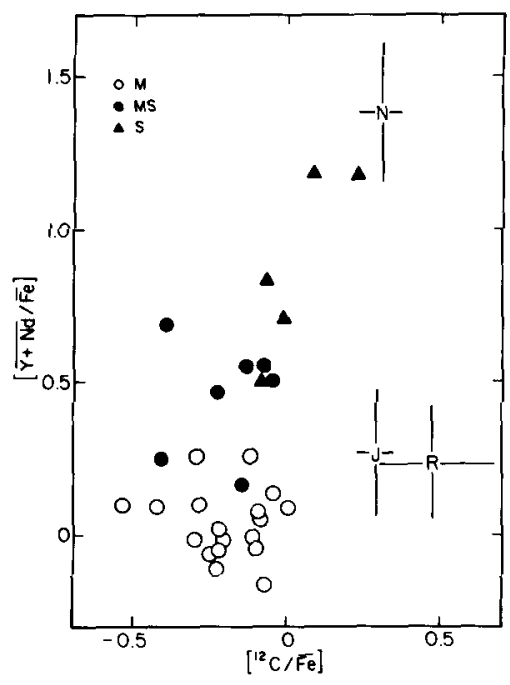

Figure 6. - The s-process overabundance $[\overline{\mathrm{Y}} \overline{+} \overline{\mathrm{N}} \mathrm{d} / \mathrm{Fe}]=\langle[\mathrm{Y} / \mathrm{Fe}]+[\mathrm{Nd} / \mathrm{Fe}]\rangle$ versus the carbon overabundance $\left[{ }^{12} \mathrm{C} / \mathrm{Fe}\right]$. Mean points for the $\mathrm{N}$-type and J-type $\mathrm{C}$ stars are obtained by combining carbon abundances from Lambert et al. (1986) with s-process abundances from Utsumi (1985). The point for the R stars is obtained from Dominy (1984).

The $\mathrm{C} / \mathrm{O}$ ratios for $\mathrm{S}$ stars span a wide range and are not clustered between $\mathrm{C} / \mathrm{O}=0.95$ to 1.0 as some interpretations of the $\mathrm{ZrO} / \mathrm{TiO}$ band intensities have suggested (see Sec. 2.1). The lower $\mathrm{C} / \mathrm{O}$ ratios obtained by us apparently reflect the fact that (i) our sample does not include "pure" S (or SC) stars for which a C/O nearer unity is expected, and (ii) the existing calibrations of the "abundance" index defined by the $\mathrm{ZrO} / \mathrm{TiO}$ bands place inappropriate stress on the role of the $\mathrm{O}-\mathrm{C}$ abundance difference and ignore the role of the $\mathrm{Zr} / \mathrm{Ti}$ abundance ratio. It should be added that our $\mathrm{C} / \mathrm{O}$ ratios have not been determined from a self-consistent analysis; the derived $\mathrm{C} / \mathrm{O}$ ratio differs from the ratio assumed in the 
construction of the model atmosphere. Elimination of this inconsistency is likely to increase our $\mathrm{C} / \mathrm{O}$ ratios by a small amount.

Comparison of the heavy (e.g., $\mathrm{Nd}$ ) and light (e.g., $\mathrm{Zr}$ ) s-process elements provides an estimate of the total exposure to neutrons; high exposure leads to a high $\mathrm{Nd} / \mathrm{Zr}$ ratio. As is common practice, I assume that the s-processed material mixed to the surface has been exposed to an exponential distribution of exposures $\rho(\tau) \sim \exp \left(-\tau / \tau_{0}\right)$ where the exposure $\tau=\int \Phi(t) d t, \Phi(t)$ is the neutron flux, and $\tau_{0}$ is the average exposure. Malaney (1988) has estimated the elemental s-process abundances for a range of $\tau_{\mathrm{o}}$ (unit: $\mathrm{mb}^{-1}$ at $\mathrm{kT}=30$ $\mathrm{keV})$. The predicted surface abundances depend on $\tau_{\mathrm{o}}$ and on the relative masses of the envelope $\left(\mathrm{M}_{\mathrm{e}}\right)$ and the s-processed material $\left(\mathrm{M}_{\mathrm{S}}\right)$. Predicted average enhancements of $\mathrm{Y}$ and $\mathrm{Zr}$ as a function of the heavy ( $\mathrm{Nd}$ ) to light ( $\mathrm{Y}$ and $\mathrm{Zr}$ ) s-process abundance ratio are shown in Figure 7 for a ratio $\mathrm{M}_{\mathrm{s}} / \mathrm{M}_{\mathrm{e}}$ up to about 0.01 and a simple two-zone model. Comparison of the observations and predictions shows that $\tau_{\mathrm{o}} \sim 0.3$ accounts for the great majority of the MS and S stars; one star (HD 35155) is remarkably Nd-rich. If the origin for the predictions is displaced to the center of gravity of the $M$ star distribution, the fit of

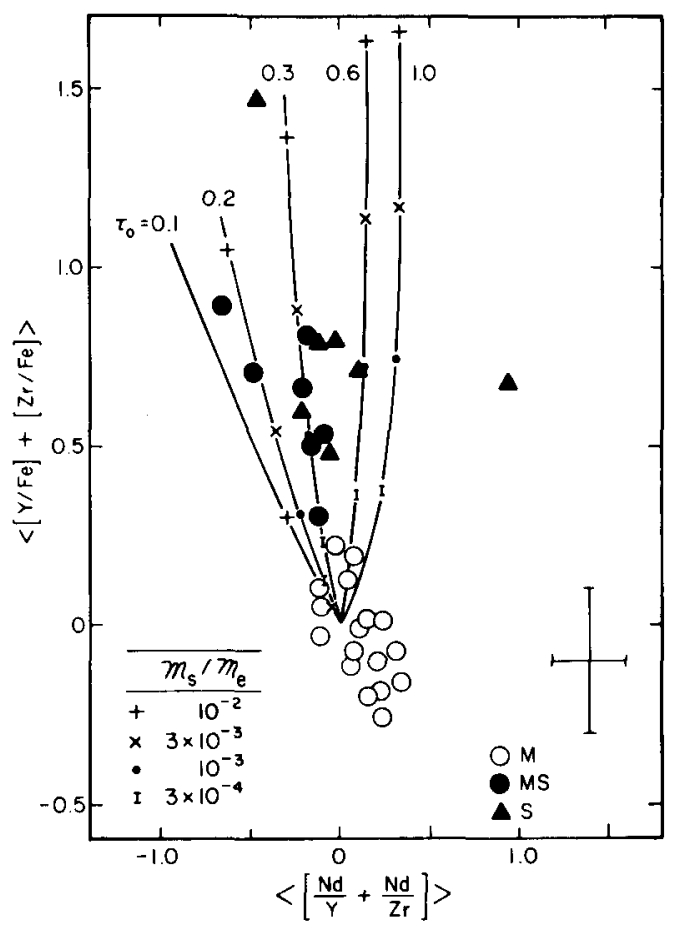

Figure 7. - The s-process overabundance $<[\mathrm{Y} / \mathrm{Fe}]+[\mathrm{Zr} / \mathrm{Fe}]>$ versus $<[\mathrm{Nd} / \mathrm{Y}]+[\mathrm{Nd} / \mathrm{Zr}]>$. Predictions are given for the 2-zone mixing described in the text. 
the $\tau_{0}=0.3$ curve to the observations is improved. The derived mixing fraction is $M_{s} / M_{e}$ $\leqslant 0.003$ with one star (TV Aur, a N-rich S star) requiring $\mathrm{M}_{\mathrm{s}} / \mathrm{M}_{\mathrm{e}} \approx 0.01$. Cool carbon stars according to Utsumi's (1985) abundance analysis fall in Figure 7 at a mean location ($0.6,+1.7)$ which is about midway between the $\tau_{0}=0.2$ and 0.3 predictions for $\mathrm{M}_{\mathrm{s}} / \mathrm{M}_{\mathrm{e}}$ 0.02 . In light of the large uncertainty $( \pm 0.4 \mathrm{dex})$ assigned to the abundances [s/Ti], this mean location derived using all four of the heavy s-elements ( $\mathrm{Ba}, \mathrm{La}, \mathrm{Nd}, \mathrm{Sm}$ ) considered by Utsumi is approximately consistent with the suggestion that the $\tau_{0}=0.3$ prediction fits the s-process enhancements of MS and S stars.

One very important conclusion is suggested by the discovery that the observed s-process enrichments correspond to synthesis at $\tau_{0} \approx 0.3$ at $\mathrm{kT}=30 \mathrm{keV}$. This estimate is identical to that derived from analysis of the solar system composition in the mass range $\mathrm{A}=90$ to 200: $\tau_{0}=0.30 \pm 0.01$ at $\mathrm{kT}=30 \mathrm{keV}$ (Walter et al. 1986; Beer 1988). We suggest that the equal $\tau_{0}$ 's, rather than being a chance coincidence, indicate that the solar s-process nuclides were synthesized in thermally pulsing AGB stars like the $M \rightarrow S \rightarrow C$ stars discussed here. Several authors have shown that such or slightly more evolved AGB stars return sufficient mass to the interstellar medium to be major contributors to its enrichment in $\mathrm{C}$ and s-process elements (Jura - this book). Since the observed s-process enrichments along the sequence $M \rightarrow S \rightarrow C$ appear to correspond to $\tau_{0} \sim 0.3$ over the entire sequence, it seems unlikely that further evolution prior to the onset of severe mass loss will change $\tau_{0}$ and so lead to the return to the interstellar medium of a non-solar mix of s-process elements.

By combining $\mathrm{M}_{\mathrm{s}} / \mathrm{M}_{\mathrm{e}}$ with the observed ${ }^{12} \mathrm{C}$ enhancements, one may estimate the mass fraction of ${ }^{12} \mathrm{C}$ in the s-processed material: $\mathrm{Z}\left({ }^{12} \mathrm{C}\right) \sim 0.5$ is a preliminary estimate from the simple two-zone model. This is satisfactorily close to the predicted $\mathrm{Z}\left({ }^{12} \mathrm{C}\right) \sim 0.25$ for shell flashes in low-mass solar-metallicity stars (Boothroyd and Sackmann 1988). The $\mathrm{He} / \mathrm{H}$ ratio at the surface is predicted to increase by no more than $1 \%$ of its initial value. Such a negligible increase is consistent with the observation that the vast majority of planetary nebulae are not enriched in He: $\log \varepsilon(\mathrm{He})=11.06 \pm 0.02$ from 39 nebulae (Aller and Keyes 1987 - N-rich PBs excluded), and $\log \varepsilon(\mathrm{He})=10.99 \pm 0.04$ for the Sun (Anders and Grevesse 1988). The question of a severe He-deficiency in cool carbon stars was addressed and dismissed by Lambert et al. (1986).

Analysis of the solar system abundances yields estimates of the neutron density and the temperature at the s-process site: $\mathrm{n}(\mathrm{n}) \simeq 1.3 \times 10^{8} \mathrm{~cm}^{-3}$ and $\mathrm{T} \simeq 2.710^{8} \mathrm{~K}$ (Beer 1988). A 
demonstration that similar conditions occur in AGB stars would strengthen our conclusion that these stars are major producers of s-process elements. The neutron density might be estimated from the ratio of the $\mathrm{Rb}$ abundance to that of neighboring elements $\mathrm{Sr}, \mathrm{Y}$, and $\mathrm{Zr}$. This method, which exploits the branch in the s-process path at ${ }^{85} \mathrm{Kr}$, has been applied to Barium K giants (Tomkin and Lambert 1983; Smith and Lambert 1984; Malaney and Lambert 1988): $2 \times 10^{7} \leqslant \mathrm{n}(\mathrm{n}) \leqslant 5 \times 10^{7} \mathrm{~cm}^{-1}$ for 4 Barium giants. We are currently investigating whether the $\mathrm{Rb}$ I 7800 and $7947 \AA$ lines are sufficiently free of blends to be useful in MS and $S$ stars. A branch at the unstable isotope ${ }^{95} \mathrm{Zr}$ provides a second densitometer: if $\mathrm{n}(\mathrm{n}) \geq 10^{10} \mathrm{~cm}^{-3}$, stable ${ }^{96} \mathrm{Zr}$ is produced. The $\mathrm{Zr}$ isotopic abundances are obtainable from analysis of $\mathrm{ZrO}$ bands; a current attempt to derive the $\mathrm{Zr}$ isotopic ratios is summarized by Smith (1988). There is no evidence in our survey of about $20 \mathrm{~S}$ stars, mostly long-period variables, for an appreciable abundance of ${ }^{96} \mathrm{Zr}$ : $\mathrm{n}(\mathrm{n})<10^{10} \mathrm{~cm}^{-3}$. As discussed by Malaney and Lambert (1988), the low neutron densities derived from the Barium giants appear to be considerably less than current predictions $\left(\mathrm{n}(\mathrm{n}) \sim 10^{9}-10^{12} \mathrm{~cm}^{-3}\right.$ ) for the He-burning shell in AGB stars. One solution to this discrepancy is to discard the mass transfer hypothesis as the origin of the Barium giants. An alternative solution is to search for modifications to AGB models that reduce the neutron density in the He-burning shell. Gallino et al. (1988) have suggested that the s-process may operate in the Heburning shell of metal-poor low-mass stars at lower neutron densities. A successful extraction of $\mathrm{Rb}$ abundances in $\mathrm{S}$ stars could test this kind of prediction.

The elemental and isotopic abundances obtainable for AGB stars do not provide a thermometer for the s-process site. The temperature, $\mathrm{T} \simeq 2.710^{8} \mathrm{~K}$, which is estimated from the solar system abundances seems inconsistent with that predicted for the active Heshell in low mass AGB stars (Boothroyd and Sackmann 1988). However, Gallino et al. (1988) remark that, according to their analysis of thermal pulses in a metal-poor low-mass star, the isotopes that serve as thermometers are synthesized during a short hot phase by a weak neutron pulse driven by the ${ }^{22} \mathrm{Ne}(\alpha, \mathrm{n})$ reaction following the major neutron release from ${ }^{13} \mathrm{C}(\alpha, \mathrm{n})$. At the rather higher temperature achieved in the shells of intermediate mass stars, the ${ }^{22} \mathrm{Ne}(\alpha, \mathrm{n})^{25} \mathrm{Mg}$ reaction replaces ${ }^{13} \mathrm{C}(\alpha, \mathrm{n})^{16} \mathrm{O}$ as the primary neutron supplier. A signature of s-processing driven by the ${ }^{22} \mathrm{Ne}$ source is an observable distortion in the Mg isotopic ratios (Truran and Iben 1977; Scalo 1978; Malaney 1987). In the Barium $\mathrm{K}, \mathrm{MS}$ and $\mathrm{S}$ stars examined to date, the $\mathrm{Mg}$ isotopes have their normal $(\approx$ solar) abundance ratios - see Tomkin and Lambert (1979), McWilliam and Lambert (1988), Malaney and Lambert (1988), and Smith and Lambert (1986). In short, the 
signature of ${ }^{22} \mathrm{Ne}(\alpha, n)$ has yet to be recognized. By default, ${ }^{13} \mathrm{C}(\alpha, n)$ is presumed to be the neutron source.

Of several chronometers provided by the s-process, technetium is the most striking - see Little-Marenin's article in this book. ${ }^{93} \mathrm{Zr}$ with a half-life of $1.5 \times 10^{6} \mathrm{yr}$ is a longer-lived chronometer. In Sec. 3.1, I mentioned Dominy and Wallerstein's (1986) discovery that o Cet is $\mathrm{Nb}$ deficient yet contains $\mathrm{Tc}$. This reduction of $\mathrm{Nb}$ is attributed to the recent $\left(<10^{5}\right.$ yr) exposure to a mild pulse of neutrons. Further investigations of abundances tied to such chronometers is likely to yield surprises.

The last few years have been exciting ones for students of PRGs. My intention in preparing this review was to illustrate the stimulating interplay between the application of "instant abundance analysis" to stars in the Magellanic Clouds and the interpretations of the comprehensive abundance analyses that are now possible for PRGs. As a result of this interplay, we may make the modest claim to understand the $M \rightarrow S \rightarrow C$ AGB stars as victims of the third dredge-up in which the products of He-shell flashes are mixed into the envelope. But much observational and theoretical work remains to be done!

Many of the results discussed in this review are the fruits of collaborations with several colleagues; I thank Drs. K. Eriksson, B. Gustafsson, K. H. Hinkle, R. A. Malaney, and V. V. Smith for their major contributions. My research is supported in part by the U. S. National Science Foundation (grant AST86-14423) and the R. A. Welch Foundation of Houston, Texas.

\section{REFERENCES}

Ake, T. B. 1979, Ap. J., 234, 538.

Aller, L. H., and Czyzak, S. J. 1983, Ap. J. Suppl., 51, 211.

Aller, L. H., and Keyes, C. D. 1987, Ap. J. Suppl., 65, 405.

Anders, E., and Grevesse, N. 1988, Geochim. Cosmochim. Acta, in press.

Becker, S. A., and Iben, I., Jr., 1979, Ap. J., 232, 831.

Beer, H. 1988, in Origin and Distribution of the Elements, ed. G. J. Mathews, (Singapore: World Scientific), p. 505.

Bessell, M. S., Wood, R. P, and Lloyd Evans, T. 1983, M.N.R.A.S., 202, 59.

Boesgaard, A. M. 1970, Ap. J., 161, 163.

Boothroyd, A. I., and Sackmann, I.-J. 1988, Ap. J., 328, 653.

Busso, M., Picchio, G., Gallino, R., and Chieffi, A. 1988, Ap. J., 326, 196.

Champagne, A. E., and Pitt, M. L. 1986, Nucl. Phys. A., 457, 367.

Cohen, J. G., Frogel, J. A., Persson, S. E., and Elias, J. H. 1981, Ap. J., 249, 481.

Cosner, K., and Truran, J. W. 1981, Ap. Space Sci., 78, 85. 
Dominy, J. F. 1984, Ap. J. Suppl., 55, 27.

Dominy, J. F., and Wallerstein, G. 1986, Ap. J., 310, 371. 1987, Ap. J., 317, 810.

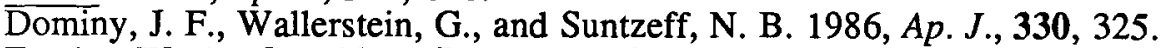

Fowler, W. A., Caughlan, G. R., and Zimmermann, B. A. 1975, Ann. Rev. Astr. Ap., 13,69 .

Frogel, J. A., and Blanco, V. M. 1984, in Observational Tests of the Stellar Evolution Theory, ed. A. Maeder and A. Renzini (Dordrecht: Reidel), p. 175.

Gallino, R., Busso, M., Picchio, G., Raiteri, C. M., and Renzini, A. 1988, preprint.

Harris, M. J., and Lambert, D. L. 1984a, Ap. J., 281, 739. 1984b, Ap. J., 285, 674.

Harris, M. J., Lambert, D. L., Hinkle, K. H., Gustafsson, B., and Eriksson, K. 1987, Ap. J., 316, 294.

Harris, M. J., Lambert, D. L., and Smith, V. V. 1985, Ap. J., 299, 375. 1988, Ap. J., 325, 768.

İben, I., Jr., 1975, Ap. J., 196, 525.

. 1976, Ap. J., 208, 165.

1977, Ap. J., 217, 788.

1981, Ap. J., 246, 278.

. 1988, in Astronomy in the Southern Hemisphere, ed. V. M. Blanco and M. Phillips, in press.

Iben, I., Jr., and Renzini, A. 1983, Ann. Rev. Astr. Ap., 21, 271.

Iben, I., Jr., and Truran, J. W. 1978, Ap. J., $220,980$.

Jorissen, A., and Mayor, M. 1988, Astr. Ap., 198, 187.

Keady, J. J., Hall, D. N. B., and Ridgway, S. T. 1988, Ap. J., in press.

Keenan, P. C. 1954, Ap. J., 120, 484.

Keenan, P. C., and Boeshaar, P. C. 1980, Ap. J. Suppl., 43, 379.

Kjaergaard, P., Gustafsson, B., Walker, G. A. H., and Hultqvist, L. 1982, Astr. Ap., $115,145$.

Lambert, D. L. 1985, in Cool Stars with Excesss of Heavy Elements, ed. M. Jaschek and P. C. Keenan (Dordrecht: Reidel).

- 1988, in The Impact of Very High S/N Spectroscopy on Stellar Physics, ed. G. Cayrel de Strobel and M. Spite (Dordrecht: Kluwer), p. 563.

Lambert, D. L., Eriksson, K., Gustafsson, B., and Hinkle, K. H. 1986, Ap. J. Suppl., $62,373$.

Lambert, D. L., and Ries, L. M. 1981, Ap. J., 248, 228.

Little, S. J., Little-Marenin, I. R., and Bauer, W. H. 1987, A. J., 94, 981.

Little-Marenin, I. R., and Little S. J. 1979, A. J., 84, 1374.

Lloyd Evans, T. 1983, M.N.R.A.S., 204, 985.

. 1984, M.N.R.A.S., 208, 447.

- 1985, in Cool Stars with Excesses of Heavy elements, ed. M. Jaschek and P. C. Keenan (Dordrecht: Reidel), p. 163.

Malaney, R. A. 1987, Ap. J., 321, 832.

. 1988, Ap. Space Sci., 137, 251.

Malaney, R. A., and Lambert, D. L. 1988, M.N.R.A.S., in press.

Mathews, G. J., Takahashi, K., Ward, R. A., and Howard, W. M. 1986, Ap. J., 302, 410.

McClure, R. D. 1983, Ap. J., 268, 264.

- 1985, in Cool Stars with Excesses of Heavy Elements, ed. M. Jaschek and P. C. Keenan (Dordrecht: Reidel), p. 315.

McClure, R. D., Fletcher, J. M., and Nemec, J. M. 1980, Ap. J. (Letters), 238, L35.

McWilliam, A., and Lambert, D. L. 1988, M.N.R.A.S., 230, 573.

Merrill, P. W. 1952, Ap. J., 116, 21.

Nussbaumer, H., Schild, H., Schmid, H. M., and Vogel, M. 1988, Astr. Ap., 198, 179. 
Pedley, J. B., and Marshall, R. M. 1983, J. Phys. Chem. Ref. Data, 12, 967.

Piccirillo, J. 1980, M.N.R.A.S., 190, 441.

Renzini, A., and Voli, M. 1981, Astr. Ap., 94, 175.

Richer, H. B. 1981, Ap. J., 243, 744.

Schatz, G. 1983, Astr. Ap., 122, 327.

Scalo, J. M. 1974, Ap. J., 194, 361. . 1976, Ap. J., 206, 474. 1978, Ap. J., 221, 627.

Scalo, J. M., Despain, K. H., and Ulrich, R. K. 1975, Ap. J., 196, 809.

Scalo, J. M., and Miller, G. E. 1981, Ap. J., 246, 251.

Scalo, J. M., and Ross, J. E. 1976, Astr. Ap. 48, 219.

Smith, V. V. 1988, in Origin and Distribution of the Elements, ed. G. J. Mathews (Singapore: World Scientific), p. 535.

Smith, V. V., and Lambert, D. L. 1984, Pub. A. S. P., 96, 226. . 1985, Ap. J., 294, 326.

- 1986, Ap. J., 311, 843.

. 1987, M.N.R.A.S., 226, 563.

, $1988 \mathrm{a}, A p . J$. , in press.

$1988 \mathrm{~b}$, in preparation.

Stephenson, C. B. 1973, Pub. Warner and Swasey Obs., 1, No. 4.

Tomkin, J., and Lambert, D. L. 1979, Ap. J., 227, 209. . 1983, Ap. J., 273, 722.

Truran, J. W., and Iben, I., Jr., 1977, Ap. J., 216, 797.

Tsuji, T. 1985, in Cool Stars with Excesses of Heavy Elements, ed. M. Jaschek and P. C. Keenan (Dordrecht: Reidel), p. 295. 1986, Astr. Ap., 158, 8.

Utsumi, K. 1985, in Cool Stars with Excesses of Heavy Elements, ed. M. Jaschek and P. C. Keenan (Dordrecht: Reidel), p. 243.

Walter, G., Beer, H., Käppeler, F., Reffo, G., and Fabbri, F. 1986, Astr. Ap., 167, 186.

Wood, P. R. 1987, in Late Stages of Stellar Evolution, ed. S. Kwok and S. R. Pottasch (Dordrecht: Reidel), p. 197.

Wood, P. R., Bessell, M. S., and Fox, M. W. 1983, Ap. J., 272, 99.

Wurm, K. 1940, Ap. J., 162, 203. 